\title{
REVIEW OF CVD SYNTHESIS OF GRAPHENE
}

\begin{abstract}
This article presents an overview of the research highlights in graphene synthesis by Chemical Vapor Deposition (CVD). We discuss the growth mechanisms mainly over transition metals and alloys (with emphasis on $\mathrm{Cu}$ and $\mathrm{Cu}$ alloys), including new developments and experiments in transfer-free graphene growth on dielectric materials. We focus on the role of the different synthesis parameters, including thermodynamic aspects of the chemical process and physical, chemical and morphological properties of substrate catalyst. We discuss the relation among these parameters and the properties of the as-grown graphene. Some important relations are reviewed and addressed to the influence of the fundamental parameters and methods on the synthesis of high quality graphene.
\end{abstract}

\section{INTRODUCTION}

Graphene, a two-dimensional, single-layer sheet of $\mathrm{sp}^{2}$ hybridized carbon atoms, has attracted worldwide attention and research interest, owing to its exceptional physical properties, such as high electronic conductivity, good thermal stability, and excellent mechanical strength [1].

Since the discovery of graphene by K. Novoselov \& A.K. Geim [2] the "top-down" exfoliation technique has been widely used to produce two-dimensional atomic crystals including not only graphene but also many other 2D materials, as BN and $\mathrm{MoS}_{2}[3]$. This process of producing graphene sheets has been found to be reliable and easy and has attracted the immediate attention of the scientific community. The best quality graphene, in terms of structural integrity, has been obtained by this method up to now. However, only flat graphene flakes (tens of microns in size) can be produced and the number of exfoliated layers is not easily controlled. As in most practical applications conceived for graphene, including microelectronics, optoelectronics (solar cells, touch screens, liquid crystal displays), graphene based batteries, supercapacitors and thermal control, large area and high quality with low structural defects graphene is needed [4] other methods should be developed. CVD synthesis route could have the answer to this complex question.

The preparation of graphite from heterogeneous catalysis on transition metals has been known for years. Independently of this, the first report on CVD synthesis of few layer graphene (FLG) was published in 2006 [5]. Since then, the CVD "bottom-up" synthesis has evolved to scalable and reliable production method of large area graphene. Synthesis of large area and high quality graphene has been demonstrated by this method [6,7]. But comparing CVD graphene properties to exfoliated graphene, the latter goes on exhibiting better quality so far.

Currently the growth and development of high quality, large-area CVD graphene on catalytic metal substrates is a topic of both fundamental and technological interest. Since the large-scale graphene films synthesized so far are typically polycrystalline, the research effort is aimed to control the domain size, the number of graphene layers, the density of grain boundaries, the defects and so on. For graphene materials to realize the promise of "graphene based applications", it is clearly necessary to solve those problems, preventing defects in fabricated devices.

There are already several and recently published comprehensive reviews dealing with theoretical properties of graphene for electronics [8] and also with future applications of graphene and graphene based materials [9-16]. The revision we are involved is aimed at people interested in practical questions in the field of synthesis and catalysis of graphene and graphene based structures. 


\section{CVD SYNTHESIS AND GROWTH OF GRAPHENE}

There exist comprehensive review articles and books dealing with generic aspects of the Chemical Vapor Deposition. Here we are only intent on describing elemental details of CVD applied to graphene synthesis. We encourage the readers to increase their knowledge with the fundamental aspects of Vapor Deposition Processes revisiting the cited references [17-23].

\subsection{BRIEF DESCRIPTION OF CVD CHEMICAL REACTIONS AND PROCESSES}

Chemical Vapor Deposition (CVD) involves the activation of gaseous reactants and the subsequent chemical reaction followed by the formation of a stable solid deposit over a suitable substrate. The energy that the chemical reaction demands can be supplied with the aid of different sources; heat, light or electric discharge are used in thermal, laser assisted or plasma assisted CVD, respectively.

The deposition process can include two types of reactions: homogeneous gas phase reactions, which occur in the gas phase, and heterogeneous chemical reactions which occur on/near the vicinity of a heated surface leading to the formation of powders or films, in each case.

Though CVD has been used to produce ultrafine powders, this review article is mainly concerned with the CVD of extremely thin graphene films. So heterogeneous chemical reactions should be favoured and homogeneous chemical reactions avoided during the designed experiments. Fig. 1 shows a schematic diagram of a typical CVD process.

a)

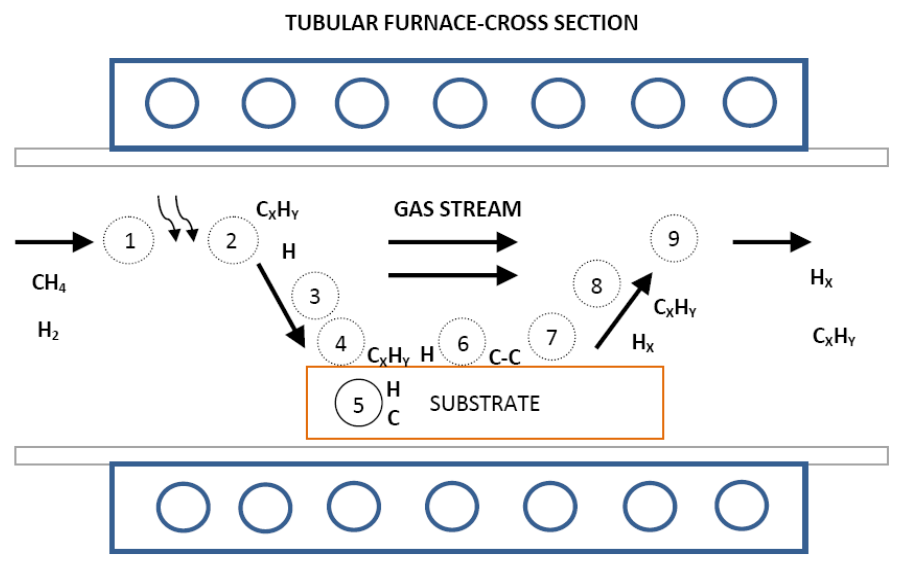

b)

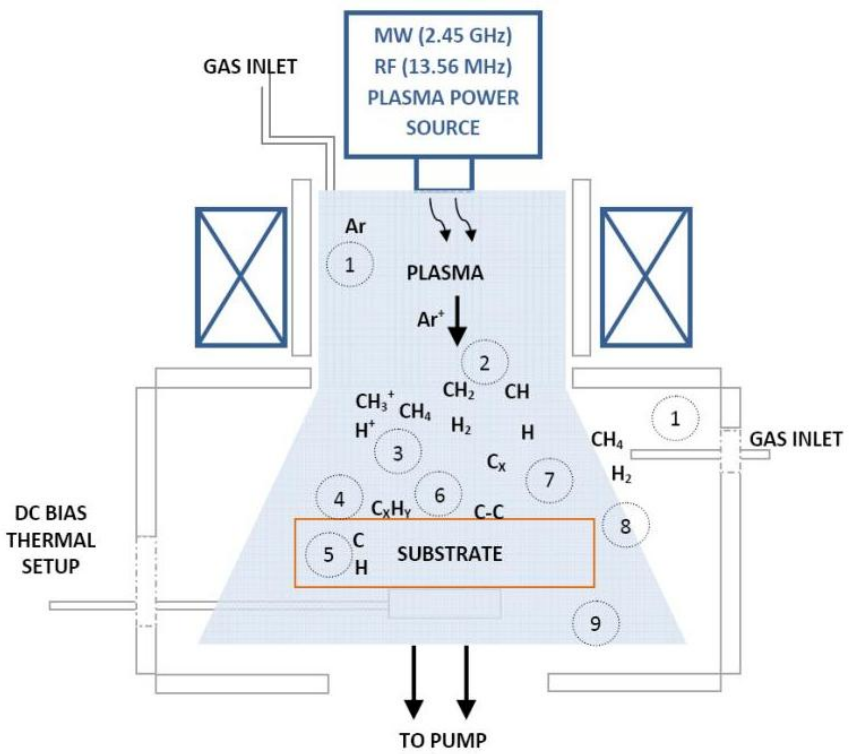

1. Transport of reactants by forced convection.

2. Thermal a) or plasma b) activation. Homogeneous gas reaction with particles and powder production should be avoided in graphene synthesis, controlling the kinetic parameters $(P, T, n)$.

3. Transport of reactants by gas diffusion from the main gas stream through the boundary layer.

4. Adsorption of reactants on the substrate surface.

5. Dissolution and bulk diffusion of species depending on the solubility and physical properties of the substrate

6. Thermal activation mediated-surface processes, including chemical decomposition (catalytic), reaction, surface migration to attachment sites (such as atomic-level steps), incorporation and other heterogeneous surface reactions. Growth of the film.

7. Desorption of byproducts from the surface.

8. Transport of byproducts by diffusion through the boundary layer and back to the main gas stream.

9. Transport of byproducts by forced convection away from the deposition region.

Fig.1. Schematic diagram of Thermal CVD a) and Plasma Assisted CVD b) process: case of graphene from $\mathrm{CH}_{4} / \mathrm{H}_{2}$ mixtures. 


\subsection{PREPARATION OF CVD-GRAPHENE.}

Chemical vapor deposition (CVD) of large-area single-layer graphene on metal films was explored widely in some respects up to now. Despite the significant progress, CVD graphene is a polycrystalline film made of micrometer to millimeter size domains. To date the graphene films grown on $\mathrm{Ni}$ foils or films did not yield uniform monolayer graphene. In most cases, a mixture of monolayer and few layers (polygraphene) was obtained. On the other hand, it was shown that $\mathrm{Cu}$ is an excellent candidate for making large area, uniform thickness (95\%) single layer graphene films due to the low solubility of $\mathrm{C}$ in $\mathrm{Cu}[6]$. It was suggested and even demonstrated that the graphene growth on $\mathrm{Cu}$ is somehow surface mediated and self-limiting.

\subsubsection{PROCESSING STEPS}

From a practical point of view, some critical steps have to be taken to get exposure of the catalyst surface to the gas precursors. One previous step is the substrate surface modification through cleaning processes, including chemical reduction inside the processing chamber. The detailed step by step process is basically as follows:

- Heating step: heating in controlled atmosphere the catalyst-substrate and gases (in hot wall reactors) up to the pre-process temperature.

- Annealing step: Maintaining the temperature and gas atmosphere so reducing the catalyst surface. This is the first chemical reaction of the whole process. It is performed to clean the catalyst surfaces and modify, as possible, the surface morphology including crystalline orientation, roughness (smoothing) and grain size of the metal catalyst. Metal evaporation should be avoided as possible.

- Growing step: Introduction of new precursors and growth of graphene over the catalyst substrate. During the growth process there are different strategies to growth the graphene film. There are onestep processes or many-step processes. During the steps is possible to modify the pressure or mix of gases, residence time, $T$, gas flow...It is important to take into account that depending on the nature of the catalyst (solubility, catalytic action and so on), the graphene may grow during this step or in the next one.

- Cooling step: After the growing step, the next step is cooling the reactor in proper atmosphere. The atmosphere commonly used is similar to that of the annealing or growing step, until the reactor temperature is under $200 \circ \mathrm{C}$ to prevent oxidation of the catalytic surface not covered or graphene functionalization with oxygen containing groups. When working with high solubility substrates, cooling step dynamics is critical to control the growth due to the solubility dependence.

- Final step: Backfill with inert gases (Ar, N) up to atmospheric pressure and open the reactor chamber.

\subsubsection{GROWTH KINETICS AND REACTION MECHANISMS}

Hydrocarbon based reactants, being methane $\left(\mathrm{CH}_{4}\right)$ the most mentioned, were commonly used as $\mathrm{C}$ source. Due to strong $\mathrm{C}-\mathrm{H}$ bonds in methane molecule $(440 \mathrm{~kJ} / \mathrm{mole})$ its thermal (non-catalytic or non plasma activated), decomposition (step 2 in fig. 1) occurs at very high temperatures ( $>1200$ ㅇ) [24]. This high temperature is not easily obtained in a typical thermal CVD setup. To reduce the temperature of methane's decomposition different transition metal catalysts (e.g., $\mathrm{Fe}, \mathrm{Co}, \mathrm{Ni}, \mathrm{Cu}$ ) were widely used. This catalytic behavior is observed when growing CVD graphene on metals at low temperatures $(\angle 900 \circ \mathrm{C})$ in a greater or lesser extent. Therefore, non catalytic activation can be considered negligible working in thermal systems [25]. On the other hand, in the case of plasma assisted CVD, the activation and decomposition of gases prior to reach the substrate is effectively performed, but surface diffusion is a thermal-mediated process and plays a fundamental role in growth kinetics. 


\section{Growth kinetics}

As the graphene synthesis process is a heterogeneous catalytical chemical reaction, the metal performs the two different roles of substrate and catalyst. Therefore, in a typical thermal catalytic CVD, the film grown over metal substrate reduces the catalytic activity due to the catalyst poisoning. This should announce the end of the reaction and the graphene film formation. If the overall process is performed on the surface (adsorption, decomposition and diffusion of molecules), monolayer graphene is preferentially grown. This is known as "self-limiting" effect and was only observed in Cu to date (and also depending on the process conditions). On Ni and other common transition metals (Co, Ru, Ir, etc.,) it was demonstrated that CVD growth of graphene occurs by carbon bulk diffusion due to the high solubility of carbon and segregation (fig.2) during cooling step. In this latter case, solid solution of a mixture of elements is formed near surface and the resulting graphene depends on the kinetic parameters selected for the synthesis. Among all the thermodynamic parameters, a fast cooling rate seems to be a critical factor to suppress the formation of multiple graphene layers $[25,26]$. More complex deposition process results when an extra gas phase activation (decomposition by plasma or very high, $>1200^{\circ} \mathrm{C}$, temperature in fig. 2 ) is performed. In this case the chemical reaction evolves to a mixture of heterogeneous catalysis and decomposition in vapour phase. Then the reaction cannot be considered as totally controlled by the catalyst.

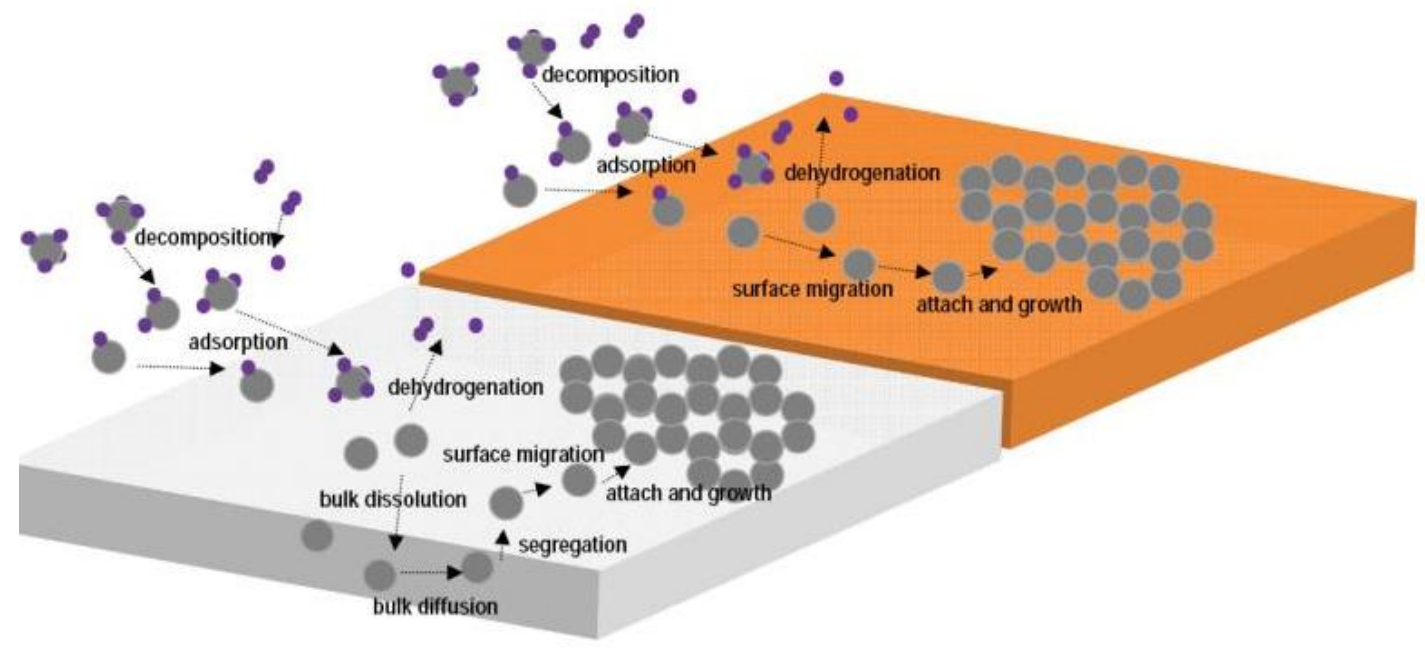

Figure 2. Growth kinetics in CVD graphene on different catalyst: Case of $\mathrm{CH}_{4}$ on $\mathrm{Ni}$ and $\mathrm{Cu}$.

The use of carbon isotope labeling technique in conjunction with Raman spectroscopic mapping $[27,28]$ demonstrated effectively different kinetic behavior of CVD growth of graphene on $\mathrm{Ni}$ and $\mathrm{Cu}$. By this technique was possible to track carbon during the growth process. The two different mechanisms of graphene growth observed on $\mathrm{Ni}$ and $\mathrm{Cu}$ can be understood from the C-metal binary phase diagram, being the most important difference that solubility of $\mathrm{C}$ in $\mathrm{Cu}$ is much lower than that in Ni. Only small amount of carbon can be dissolved on $\mathrm{Cu}$. The source of $\mathrm{C}$ is mainly $\mathrm{CH}_{4}$ that is catalytically decomposed (dehydrogenated in fig.2) on the $\mathrm{Cu}$ surface. This route facilitates surface migration and monolayer graphene growth. Experiments with high temperature cycles performed on graphene films of approximately 0.5 monolayer coverage on $\mathrm{Cu}$ while continuously imaged using LEEM (Low-Energy Electron Microscopy) confirmed this demonstration [29]. No C precipitation or island growth was observed during cooling in agreement with preliminary reports, suggesting that the process is confined to the surface, with negligible dissolution and precipitation of $C$ from the substrate.

In contrast, $\mathrm{Ni}$ can dissolve much more carbon atoms. The graphene growth becomes mainly from the precipitation during the cool-down of the process and "polygraphene" was detected in most cases. One 
opportunity for $\mathrm{Ni}$ is that the solubility and precipitation process can be controlled to some extent with the annealing, growing and cooling rates.

It is worthy of mention that, in the case of $\mathrm{Cu}$ it was published that not always the result is a graphene monolayer, there can also be a small fraction of flakes (few layers) stacked on the graphene film. To dispel doubts, termination of flake growth due to full coverage of $\mathrm{Cu}$ surface with graphene was observed, that suggests that the carbon species for flake growth had similar origin as those for the first graphene layer. $[30,31]$ One possible explanation for these small multilayer areas could be that the nature, composition and morphology of nucleation centers had an important role in the first stage of nucleation. Also is important to note that for the graphene layer in contact with the metal, the edge growth rate could be faster than the growth rate of the second and subsequent layers that may be attributed to a more difficult access of the species to upper- or lower- layers.

\section{Reaction mechanisms}

One explanation of reaction mechanism depending on the nature of the catalyst was already proposed, based on the spectroscopic elipsometry experiments [32]. Primarily, the difference in the growth kinetics and mechanism between $\mathrm{Ni}$ and $\mathrm{Cu}$ was ascribed to the really different carbon solubility in Cu compared to $\mathrm{Ni}$. But the mechanism shows more complex differences.

\section{Graphene synthesis: reaction mechanisms}

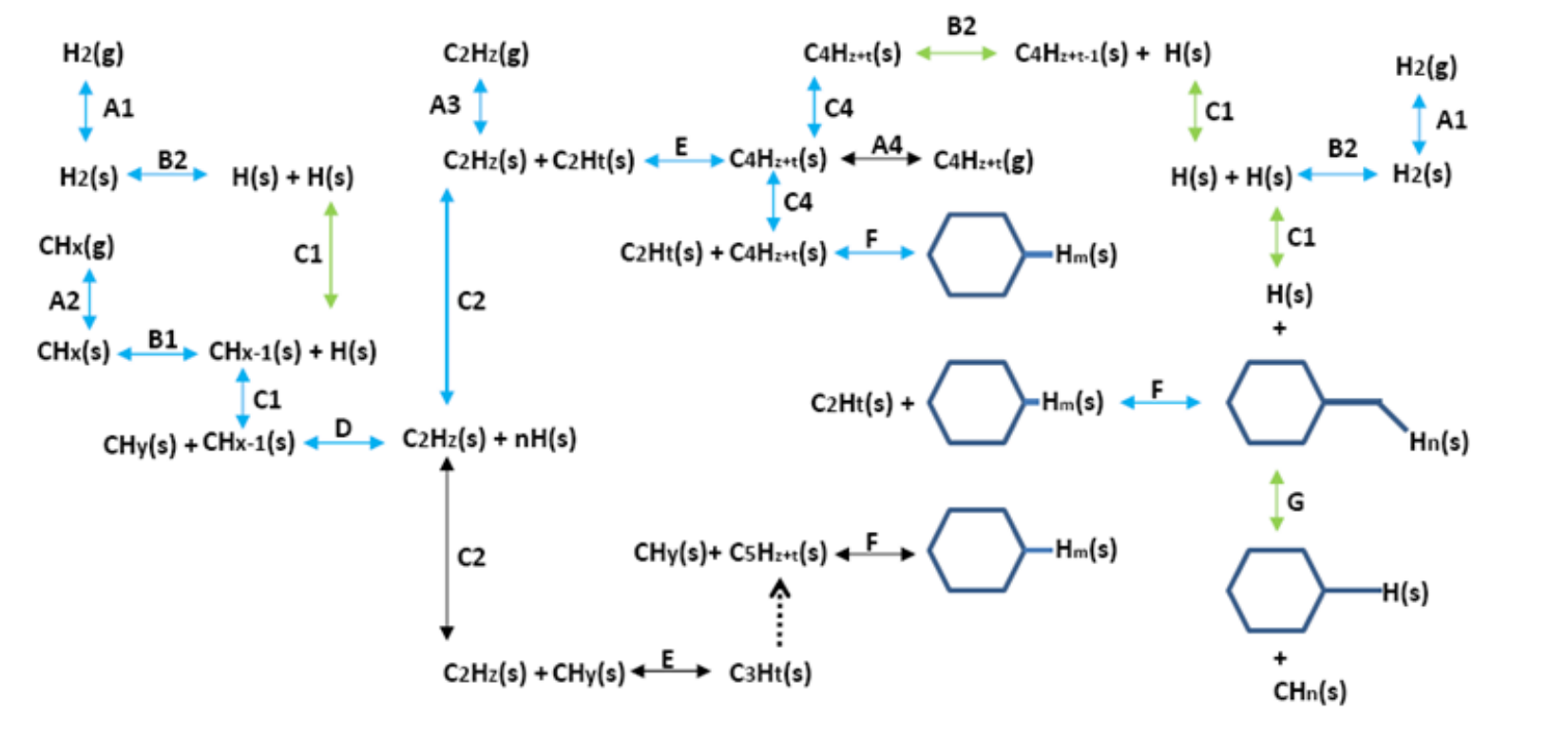

Figure 3. Reaction mechanisms in CVD graphene on transition metal catalyst. More probable (but not unique) reaction mechanisms in blue arrows and $\mathbf{z}, \mathbf{t}, \mathbf{m}=\mathbf{0}$. Reaction type $\mathbf{A}$ : adsorption-desorption. $\mathbf{A} \mathbf{1}, \mathbf{A} \mathbf{3}$ desorption favoured by hydrogen saturation on surface (from precursors). B: dehydrogenation-hydrogenation. Probable source for hydrogenation is molecular $\mathrm{H}_{2}$ precursor. C: surface diffusion or migration, more favorable for dimmers (C2). Dimmers with high hydrogen saturation probably suffer desorption or cracking. D: dimerization with or without simultaneous dehydrogenation - (cracking) decomposition [v]. Dimers with hydrogen are not stable at high temperatures. E: polymerization- (cracking) decomposition. F: aromatization decomposition. More stable arm-chair edges. G: decomposition of aromatics (hydrogen attack). Reactions with hydrogen in green arrows.

A general picture of the reaction mechanisms during synthesis is shown in fig. 3. During annealing step, the catalyst surface is reduced by molecular hydrogen. At the end of this process the nude metal surface gets also exposed to hydrogen so, the first step to be considered should be the dissociative chemisorption 
of $\mathrm{H}_{2}$ on the metal surface, reaction (B2). At typical conditions of graphene synthesis, this process takes place on $\mathrm{Cu}$ and $\mathrm{Ni}$ surfaces with different trends. In the case of $\mathrm{Ni}$ is more probable for hydrogen to recombine and desorb from surface (reaction $\mathrm{B} 2, \mathrm{~A} 1$ ) but this is not directly applicable for Cu that exhibits a much more hydrogen solubility $[32,47,48]$. In this case, saturation would be necessary to desorb molecular hydrogen from $\mathrm{Cu}$ surface. Therefore, before exposure of the catalyst to hydrocarbons a surface and/or subsurface partially covered with atomic hydrogen could be the starting point [46].

After exposure to hydrocarbons (diluted in molecular hydrogen in most cases) the next step to discuss is the competitive process between the dissociative chemisorption of $\mathrm{H}_{2}$ and the physical adsorption and dehydrogenation of $\mathrm{CH}_{4}$ on available surface sites (s) of the catalyst ( $\mathrm{Ni}$ or $\mathrm{Cu}$ ), according to reactions (A1B2) and (A2-B1), respectively.

During next steps $\mathrm{CH}_{\mathrm{x}}\left(\mathrm{CH}_{4}\right)$ catalytic decomposition (dehydrogenation in fig 2, and reaction $\mathrm{B} 1$ in fig 3) takes place on the metal surface. The precise moment when the precursor dehydrogenation is completed remains an open question. According to theoretical calculations based on density functional theory (DFT) $[35,50,36]$ dehydrogenation reactions are probable to take place up to $x=2$ (fig 3 ) in the case of $\mathrm{Cu}$, being the $\mathrm{CH}$ monomer dissociation the rate limiting step, difficult to complete. On the other hand, complete monomer dehydrogenation and carbon bulk diffusion is expected in the case of high solubility metals ( $\mathrm{Ni}$ ) even though $\mathrm{CH}$ dissociation has high activation barrier [49] even on steps [50] at the process temperatures. These monomers on $\mathrm{Cu}$ continue their path towards graphene nucleation, being dimmer formation with simultaneous dehydrogenation a favorable reaction from an energetic point or view (reaction D, fig 3). According to first-principles calculations within density functional theory [37] C=C dimmers are stable on all sites on $\mathrm{Cu}$ surface. Moreover carbon dimmers containing hydrogen are very unfavorable on surface with low adsorption energies [39, 42, 43] even in defects [44] and desorb or immediately decompose even at very low temperatures $[40,45,53]$ as demonstrated in TemperatureProgrammed Desorption (TPD) and Thermal Desorption Spectroscopy (TDS) experiments [52]. Complementary studies of acetylene $(\mathrm{CH} \equiv \mathrm{CH})$ aromatization over transition metals also demonstrated that the benzene ring is not stable at the metallic surface [41]. Therefore, in the case of $\mathrm{Cu}$, the reaction $\mathrm{D}$ with $\mathrm{z}=0$ should be considered in carbon deposition, being this moment the most probable to complete the dehydrogenation and the formation of $\mathrm{C}=\mathrm{C}$ bonds with $\mathrm{sp}^{2}$ hybridization. Other routes that include carbon dimmers, trimmers and tetramers as a result of carbon atom by atom incorporation (reactions $E, F$ in black fig 3) were also studied by DFT methods [51]. Important activation barriers were involved in this reaction path due to the formation of bridging-metal (BM) structures with energy barriers higher than dimmers diffusion barriers and subsequent aromatization. To clarify the nucleation model on $\mathrm{Cu}$ (111) Riikonen et al., [38] emphasized the role of mobile carbon dimmers during the growth of graphene on $\mathrm{Cu}$. Several competing processes were taken into account, namely, (1) dimmer formation, (2) dimmer migration, (3) back dissociation of dimmers into individual atoms, (4) migration of carbon along the surface and (5) migration of carbon atoms deeper into the bulk. On the basis of theoretical calculations, the processes (1) and (2) were found to be dominant. In detail, it was observed that the formation of carbon dimmers was exothermic and that the migration barrier for the dimmer to move on the $\mathrm{Cu}(111)$ surface was small $(\mathrm{Ea}=0.27 \mathrm{eV})$. Once the dimmer was formed, it cost more energy to dissociate the dimmer than it to migrate around the surface. They also had shown how migrating dimmers could form larger graphitic structures on the $\mathrm{Cu}(111)$ facet. Interplay of the substrate electronic states with the dimmer $\sigma_{p}$-bonding orbital was observed: there should be an electronic factor that stabilizes the internal $\mathrm{C}-\mathrm{C}$ bonding of the carbon dimmer, while at the same time reducing its interaction with copper. This could be an important mechanism in driving the formation of stable and mobile graphitic rings and fragments on copper. Suitable selection of thermodynamic parameters during synthesis is crucial, that allow these surface carbon species to have lower chemical potentials than carbon in gas phase. Only in this way carbon graphitic rings could be stable at surface and could grow into larger graphitic structures up to graphene formation [35]. Once the nucleated graphene structure is stable at surface, further growth can be performed by attachment of carbon species onto graphene edges. Theoretical analysis of graphene edge reconstructions showed that $\mathrm{C}$ insertion into the front of a growing graphene patch must depend on the edge configuration. On $\mathrm{Cu}$ (111), a carbon dimmer has lower formation energy than a 
monomer. Armchair (ac) edges are also more stable than other edge possible configurations, and thus, the addition of $\mathrm{C}$ should occur preferentially (but not only) in the form of dimmers to armchair edges. After a C2 dimmer diffuses to an armchair site and forms a hexagon on the pristine ac edge, the second hexagon then forms at an adjacent ac site by insertion of another C2 dimmer [54].

\section{TOWARDS HIGH QUALITY GRAPHENE: EFFECT OF EXPERIMENTAL PARAMETERS}

CVD chemical reactions, graphene synthesis steps and growth kinetics aspects, have just been reviewed, but growth kinetics and reaction paths are not completely known, and more complementary experimental work should be performed to get to know the actual precursor decomposition routes in each case. The detailed knowledge of the growth kinetics and the reaction paths have proven to be of great importance when working on CVD synthesis. In this section, the fundamental factors that could have great influence on high quality synthesis of monolayer, bilayer or few-layer graphene films will be discussed. The correct synthesis parameters should be selected to achieve high quality graphene.

\subsection{TYPE OF PRECURSOR MATERIAL.}

Gas, liquid and solid precursors were used for graphene synthesis, [55] being mainly hydrocarbons and polymers ( $\mathrm{C}$ and $\mathrm{H}$ based compounds). The fundamental question to bear in mind is that they are all suppliers of $\mathrm{C}$ and just before reaching the surface they are always in the gas phase. Therefore, a critical parameter in graphene synthesis is the energy needed to perform the whole process depending on the type of precursor. [36][34]. One way to reduce this energy demand could be the use of low $\mathrm{C}-\mathrm{H}$ bond energy precursors, being other way the use of catalysts, not discussed in this section. Accordingly, a key parameter to take into account should be the dehydrogenation energy of the precursor $\left(\mathrm{CH}_{\mathrm{x}}\right.$ to $\left.\mathrm{CH}_{\mathrm{x}-1}\right)$. This is a highly endothermic process in the gas phase although on the metal surface, there is a significant reduction of the energy required, owing to the presence of strong $\mathrm{M}-\mathrm{CH}_{x-1}$ and $\mathrm{M}-\mathrm{H}$ interactions. [36]

As we have seen above, the decomposition (dehydrogenation) in gas phase can be performed by high temperature or plasma assisted processes. On the other hand, when the species are adsorbed the decomposition is a catalytic process. The gas phase decomposition, always performed before adsorption, was widely used in typical reactions in horizontal CVD quartz hot-reactors. But in some cases, as in $\mathrm{CH}_{4}$ graphene synthesis, the effect of the thermal heating of the gas is negligible, so thermo-catalytic decomposition on metal surface is crucial. Later it will be discussed that currently in experimental work, plasma CVD (activation of gases with electromagnetic energy) combined with thermal heating of the catalysts is being widely used. Therefore, there are two possible routes in synthesis: gas pre-activationdissociation before reaching the substrate or only thermo-catalytic decomposition (see figure 1).

\subsubsection{GASES}

\section{Hydrocarbons}

Methane $\left(\mathrm{CH}_{4}\right)$, ethylene $\left(\mathrm{CH}_{2}=\mathrm{CH}_{2}\right)$ and acetylene $\left(\mathrm{C}_{2} \mathrm{H}_{2}\right)$ being the dehydrogenation energies of $\mathrm{CH}_{4}(410$ $\mathrm{kJ} / \mathrm{mol}=98.4 \mathrm{Kcal} / \mathrm{mol}),\left(\mathrm{CH}_{2}=\mathrm{CH}_{2}\right)(443 \mathrm{~kJ} / \mathrm{mol}=106.32 \mathrm{Kcal} / \mathrm{mol})$ and $\left(\mathrm{C}_{2} \mathrm{H}_{2}\right)(506 \mathrm{~kJ} / \mathrm{mol}=121.44 \mathrm{Kcal} / \mathrm{mol})$ [56] were used as typical gaseous carbon precursors. The $\mathrm{C}-\mathrm{H}$ bond energy is a key parameter to control the decomposition temperature and required energy. Methane, the most used, is highly stable saturated molecule so the dehydrogenation in the gas phase of $\mathrm{CH}_{\mathrm{x}}$ to $\mathrm{CH}_{x-1}$ is highly endothermic, the calculated values being $\mathrm{CH}_{3}-\mathrm{H}, 4.85 \mathrm{Ev} ; \mathrm{CH}_{2}-\mathrm{H}, 5.13 \mathrm{eV} ; \mathrm{CH}-\mathrm{H}, 4.93 \mathrm{eV}$ ); $\mathrm{C}-\mathrm{H}, 3.72 \mathrm{eV}$ [36]. On the metal surfaces, there is a significant reduction in these values and theoretical calculations explained that over some transition metals even exothermic dehydrogenation processes could take place. 


\section{Hydrogen content}

Hydrogen $\left(\mathrm{H}_{2}\right)$, were widely used in the cleaning and crystallization of the metallic substrates (annealing step), via oxygen reduction ( $\mathrm{Mx}-\mathrm{O}, \mathrm{M}-\mathrm{Ox}$, oxides are always present in the catalyst surface). Molecular Hydrogen $\left(\mathrm{H}_{2}\right)$, used as diluent gas of the carbon precursor (typically $\mathrm{CH}_{4}$, various ratios of $\mathrm{CH}_{4}-\mathrm{H}_{2}$ have been reported in the literature) undoubtedly has to have a role in the graphene CVD growth. [57] As we have discussed in previous sections, its interaction with the substrate could affect the subsequent $\mathrm{CH}_{4}$ chemisorption kinetics [32], i.e.,

(i) $\mathrm{H}_{2}$ and/or atomic $\mathrm{H}$ could diffuse into the catalyst depending on the solubility (metals of high hydrogen solubility and low surface diffusion) and compete with $\mathrm{CH}_{4}$ for the initial physical adsorption;

(ii) atomic $\mathrm{H}$ could create sites for sticking hydrocarbon and carbon radicals on the surface by subsequent $\mathrm{H}$-abstraction reactions, removing hydrogen from the surface;

(iii) hydrogen could passivate defects and grain boundaries, that were believed nucleation sites;

(iv) atomic hydrogen could be active in the competition of $\mathrm{CHx}$ deposition or $\mathrm{C}$-etching, and

(v) it could play an important role in the C sp3 - sp2 transition.

Indication of the important role of hydrogen in determining the graphene growth kinetics and in limiting the graphene thickness comes from previous observations such as that when the fraction of $\mathrm{CH}_{4}$ with respect to $\mathrm{H}_{2}$ is increased, the graphene growth on $\mathrm{Cu}$ is no longer self-limiting [58]. But the contrary has also been published (depending on other synthesis conditions) [59].

Another question that it is worthy to emphasize is the differences detected on the role played by hydrogen during the pretreatment and the whole process depending on the metal used ( $\mathrm{Cu}$ or $\mathrm{Ni}$ ). As an example, a reversible phenomenon with respect to hydrogen for $\mathrm{Cu}$ was observed. Hydrogen readily bulk-diffuses into $\mathrm{Cu}$, and it out-diffuses to surface when hydrogen is turned off and pressure decreased but it does not desorb from surface, unlike Ni. This is consistent with different diffusion coefficients in $\mathrm{Cu}$ and $\mathrm{Ni}$. At the typical growth temperature of graphene by CVD of approximately $900 \stackrel{\circ}{\circ}$, the in-diffusion coefficient of hydrogen in $\mathrm{Cu}$ is approximately one order of magnitude higher than for $\mathrm{Ni}$, implying a lower hydrogen solubility in $\mathrm{Ni}$ than in $\mathrm{Cu}$ [33]. So the reaction (0) below is highly favored for $\mathrm{Ni}$ but not for $\mathrm{Cu}$. So the role of hydrogen and its benefits or damages during growth remains an open question.

$$
H(s)+H(s)-H_{2}(g)+2(s)(0)
$$

\subsubsection{LIQUIDS}

A recent publication reported the use of toluene and LPCVD to grow continuous monolayer graphene films at $500^{\circ} \mathrm{C}$ to $600^{\circ} \mathrm{C}$ on flat and electropolished Cu foils (after $980^{\circ} \mathrm{C}$ annealing) [64]. The consideration that motivated the choice of toluene, along with the fact that it is considerably less toxic than others as benzene, was its actually weak bonds. In this work, particularly it should be highlighted the results about the rectangular shape graphene domains. The authors related it to the hydrogen partial pressure of the reactor. Comparing the partial pressure of hydrogen reported in the literature and the partial pressure of these results, the higher the partial pressure of hydrogen, the easier the domain shapes tend to be equiaxed shape due to more sharp edges and corners of the graphene flakes etched. This means that sharp four or six lobe domains can grow under lower partial pressure of hydrogen and the hexagonal domains under higher hydrogen partial pressure. The partial pressure of hydrogen for growing rectangular domains that were observed was between them. 


\subsubsection{SOLIDS}

There were published many experimental works dealing with the use of solid precursors in graphene synthesis. It was demonstrated that large area, high quality graphene can be grown from solids. Among these precursors, there are polymer films, small molecules, evaporated solids etc... Interestingly, even food, insects, and waste were used as solid carbon source to generate high-quality monolayer graphene. [60]

One first approach was the use of solid polymers (polystyrene) as the carbon source because of its relatively weak $\mathrm{C}-\mathrm{H}$ bonds and low decomposition temperature [61]. The $\mathrm{C}-\mathrm{H}$ bond in polystyrene is comparatively weak, with a bond energy in between $292-305 \mathrm{~kJ} / \mathrm{mol}$, much lower than that in typical gaseous carbon precursors such as methane $(410 \mathrm{~kJ} / \mathrm{mol})$, ethylene $(443 \mathrm{~kJ} / \mathrm{mol})$ and ethyne $(506 \mathrm{~kJ} / \mathrm{mol})$. Varying $\mathrm{H}_{2}$ flow and heating polystyrene up to $280^{\circ} \mathrm{C}$, it is possible to grow hexagonal graphene domains (up to $1.2 \mathrm{~mm}$ ) with low nucleation density $100 \mathrm{n} / \mathrm{cm}^{2}$ in AP (Atmospheric Pressure) CVD.

Other example dealing with spin-coated poly methyl methacrylate (PMMA) and small molecules (fluorene $(\mathrm{C} 13 \mathrm{H} 10)$ and sucrose, table sugar, $\mathrm{C} 12 \mathrm{H} 22 \mathrm{O} 11$ ) deposited on $\mathrm{Cu}$ at temperatures from $800 \circ \mathrm{C}$ to $1000{ }^{\circ} \mathrm{C}$ were reported under LP (Low Pressure) CVD conditions [55]. It was found that the thickness of PMMAderived graphene can be controlled-to give a monolayer, a bilayer, or a few layers-by changing the diluent gas flow rate. In the case of small molecules, although containing potential topological defect generators (the five-membered ring in fluorene) or high concentrations of heteroatoms (oxygen in sucrose), they also produce high quality pristine graphene [55]. Other substrates-such as $\mathrm{Ni}, \mathrm{Si}(100)$ with native oxide, and 200-nm-thick $\mathrm{SiO}_{2}$ thermally grown-were also tested. The Raman spectra confirm that $\mathrm{Ni}$ is an efficient catalytic substrate that converts PMMA into highly crystalline graphene. Under the same growth conditions, neither graphene nor amorphous carbon was obtained on $\mathrm{Si}$ or $\mathrm{SiO}_{2}$ substrates.

Also Hofrichter et al. published the fabrication of graphene on silicon dioxide by solid-state dissolution of an overlying stack of a silicon carbide layer $(50 \mathrm{~nm})$ and a nickel thin film $(500 \mathrm{~nm})$ [62]. The carbon dissolves in the nickel by rapid thermal annealing. Upon cooling, the carbon segregates to the nickel surface forming a graphene layer over the entire nickel surface. By wet etching of the nickel layer, the graphene layer was allowed to settle on the original substrate. The $\mathrm{SiO}_{2} / \mathrm{Si}$ substrate was annealed at $1100^{\circ} \mathrm{C}, 30$ seconds in AP of Nitrogen. When cooled, the carbon segregates to the interface forming graphene layer resulting in few layer and monolayer mix (polygraphene). Further optimization to obtain larger monolayer coverage should be possible via carefully tuning the ratio of $\mathrm{SiC}$ to $\mathrm{Ni}$ film thickness in conjunction with an optimized cooling rate [63].

Homogeneous single-layer graphene can be growth by simply annealing crystalline $\mathrm{Cu}$ (111)/c-plane sapphire at 900 and $1000{ }^{\circ} \mathrm{C}$, due to graphitization of amorphous carbon previously deposited on the surface or coming from furnace walls desorption [65]. But the published results confirm that only if sufficient carbon source is supplied, graphene can be formed, irrespective of growth temperature (800$1000^{\circ} \mathrm{C}$ ) and nature of the metal, suggesting that the carbon supply is an important influencing factor of graphene nucleation. So, surface induced graphitization has not proved suitable for synthesis of high quality material.

\subsection{SUBSTRATE}

Since the metal exert a key role, as catalyst, in the formation of graphene layers, in this section we take a look at the critical issues concerning the substrate material. Chemical and physical properties, crystallography and morphology of the catalyst are reviewed. We address the differences due to the metallic or dielectric character of the substrates. 


\subsubsection{MATERIALS}

\section{Transition metals}

The formation of few layered graphene resulting from industrial heterogeneous catalysis on transition metal surfaces has been known for years. The catalytic power of transition metals and some of their compounds is well known and arises from their partially filled d-orbitals or from de formation of intermediate compounds that favors the reactivity of the precursor gases. Therefore, catalysis by metals results from their ability to provide low activation energy pathways for reactions either by the facile change of oxidation states or formation of intermediate compounds.

The CVD method on transition metals such as $\mathrm{Ni}$ [66], Pd [67], Ru [68,70], Ir [69,70] and Cu[6] foils or evaporated films, revealed that the properties of the as-grown graphene films such as quality, continuity, and layer number distribution are dependent on the catalyst used. The different catalytic activity and solubility were detected to lead to different growth mechanisms.

Catalytic activity is related to decomposition of hydrocarbons on metals, which produces active carbon species and is a critical step lowering the activation energy for decomposition of precursor gases. As an example, the fact that graphene can grow on $\mathrm{Pt}$ at a relatively low temperature $\left(750{ }^{\circ} \mathrm{C}\right)$ indicates that $\mathrm{Pt}$ has a stronger catalytic ability for $\mathrm{CH}_{4}$ dissociation than $\mathrm{Cu}$ [71], which is consistent with reported theoretical calculations [36]. Pt also has a much stronger catalytic ability for $\mathrm{H}_{2}$ dissociation to form active atomic $\mathrm{H}$. The lower activity of $\mathrm{Cu}$ also compared to $\mathrm{Ni}$ and other transition metals in the catalytic dissociative chemisorptions of $\mathrm{CH}_{4}$ can be rationalized considering that it occurs by the electron transfer from the $\mathrm{C}-\mathrm{H}$ bonds to the $3 \mathrm{~d}$ orbitals of the catalysts, with $\mathrm{Ni}$ having two $3 \mathrm{~d}$ unpaired electrons and $\mathrm{Cu}$ having only one unpaired electron available for the interaction (Cu has electron configuration $[\mathrm{Ar}] 3 \mathrm{~d}^{10} 4 \mathrm{~s}^{1}$, since an electron pass from the $4 \mathrm{~d}$-orbital to $3 \mathrm{~d}$ to generate a filled $3 \mathrm{~d}$ electron shell, which is the most stable configuration) [32]. Copper has not been observed to form any carbide phases, so the low reactivity with carbon could be attributed to the fact that copper has a filled $3 \mathrm{~d}$-electron shell, the most stable configuration (along with the half filling $3 \mathrm{~d}^{5}$ ) because the electron distribution is symmetrical which minimizes reciprocal repulsions [30].

The carbon solubility also revealed as a key parameter to control the growth of graphene over metals. Different growth kinetics were proposed depending on the solubility of the catalyst. Also the growth conditions determine the deposition mechanism defining the morphology (domain size and boundaries) and thickness of the graphene films. $\mathrm{Cu}$, e.g., has very low carbon solubility compared to $\mathrm{Co}$ and $\mathrm{Ni}$ (0.001- 0.008 weight \% at 1084 ㅇ C for $\mathrm{Cu}, 0.6$ weight \% for $\mathrm{Ni}$ at 1326 으, and $0.9 \%$ weight for $\mathrm{Co}$ at 1320 으).

As a result of the low solubility and low catalytic activity, $\mathrm{Cu}$ can form only soft bonds with carbon via charge transfer from the $\pi$ electrons in the $\mathrm{sp}^{2}$ hybridized carbon to the empty $4 \mathrm{~s}$ states of copper [14, 71,72]. This combination of very low affinity between carbon and copper along with the ability to form intermediate soft bonds could facilitate graphitic carbon formation. As the $3 d^{7}$ and $3 d^{8}$ orbitals of Co and $\mathrm{Ni}$ are between the most unstable electronic configuration $(\mathrm{Fe})$ and the most stable one $(\mathrm{Cu})$, it could be postulated that the most suitable catalysts for graphitic carbon formation are those transition metals that have low affinity towards carbon but that are still able to stabilize carbon on their surfaces by forming weak bonds [14].

Previous results of growth on polycrystalline $\mathrm{Ni}[66,74,75]$ and $\mathrm{Cu}[6]$ substrates triggered interest in graphene synthesis by CVD for large area deposition. In the case of $\mathrm{Ni}$, the fundamental limitation was that polygraphene was mainly obtained. This lack of control over the number of layers was partially attributed to the fact that the segregation of carbon from the metal carbide upon cooling due to high solubility $(\sim 0.6 \%)$, occured at different rates within the Ni grains and at the grain boundaries [73]. There 
are some reports [63] of graphene films composed mostly of one or two layers of graphene grown by controlled carbon precipitation on the surface of polycrystalline Ni thin films during atmospheric (CVD). Controlling both the methane concentration and the substrate cooling rate during growth can significantly improve the thickness uniformity.

In contrast to $\mathrm{Ni}$, uniform deposition of high quality, single layered graphene over large areas was recently achieved on polycrystalline copper foils [6]. The initial [6] and subsequent follow-on [7] studies demonstrated the growth of single layered graphene over areas as large as 30 -inches with $95 \%$ coverage of monolayer graphene. CVD growth of graphene on $\mathrm{Cu}$ is generally attributed to thermal decomposition of hydrocarbons on the surface and the subsequent surface diffusion of carbon atoms due to the low solubility of carbon ( $<0.001$ at.\%) in Cu. Furthermore, thin copper foils are inexpensive and can be easily etched with solvents available in most laboratories so that transfer onto desired substrates can be readily achieved.

A recent publication [71] claimed the growth of millimeter-sized hexagonal single-crystal graphene flakes and graphene films on single crystal and polycrystalline Pt by ambient-pressure CVD. In this case, singlecrystal Pt substrate showed similar growth behavior for graphene as that on polycrystalline Pt. Solubility of carbon in $\mathrm{Pt}$ is high, $0.9 \%$ in weight, so growth temperature of lower than $800{ }^{\circ} \mathrm{C}$ was enough for graphene nucleation. Low $\mathrm{CH}_{4}$ concentration was used because the size of the graphene grains was related with this parameter. Single crystal grains of a perfect hexagon shape with very smooth edges and lateral size of up to $1.3 \mathrm{~mm}$ were obtained at 1040 ㅇ. .

Compared to $\mathrm{Cu}$ or $\mathrm{Ni}$ catalyst systems, $\mathrm{Fe}$ is favorable in terms of low cost. However, there were only a few studies in this area maybe due to the complex $\mathrm{Fe}-\mathrm{C}$ phase diagram that also offers great versatility [76]. Lowering Ta below 912 으 $\mathrm{C}$ results in phase transformation of the Fe-C binary mixture to bodycentered cubic alfa-ferrite and a decrease in the solubility of $C$, due to the eutectic phase formation at 727 - $C$ with a very small solubility of about 0.022 wt\%. In this case, few-layer graphene were grown by APCVD with $\mathrm{CH}_{4}$. The layer number distribution were tuned by varying the procedure of heating/cooling temperature, cooling rate and $\mathrm{CH}_{4}$ flow rate.

Metal-catalyzed crystallization of a-C to graphene by thermal annealing (deposition of a layer of a-C, 2.5$40 \mathrm{~nm}$ thick by electron-beam evaporation on $\mathrm{Si}_{-} \mathrm{SiO}_{2}$ substrates, followed by nickel or cobalt metal thin film, $100-300 \mathrm{~nm}$ deposition) was also performed [77]. These samples were annealed at $650-950^{\circ} \mathrm{C}$ using a tube furnace under argon flow.

In conclusion, up to now the best approach to control the number of layers via metal catalysis was the monolayer growth on very low solubility metal, as $\mathrm{Cu}$, since it was quite difficult to control the growth of monolayer graphene via fast cooling processes on high solubility metals ( $\mathrm{Ni}$ ). It was also observed a lack of control in growing bilayer graphene, which is actually important because bilayer films seem to be the most useful in electronic applications.

\section{Metal Alloys}

\section{$\mathrm{Cu}-\mathrm{Ni}$}

Recently, more experimental efforts were made with the intention of controlling the number of layers of nucleated graphene films that would be a real breakthrough. Thin Ni films and fast-cooling processes were used to hinder carbon precipitation. Not uniform graphene but polygraphene with only monolayer regions and a wide range of thicknesses was grown by these methods. It was widely observed that monolayer graphene grows on the "flat" dimension boundaries of the metal grains while multilayer 
graphene $(n>3)$ preferentially forms at the metal grain boundaries. This lack of control was the main reason to study the growth of graphene on metal alloys.

Since $\mathrm{Cu}$ and $\mathrm{Ni}$ are well-known binary isomorphous systems, $\mathrm{Cu}-\mathrm{Ni}$ alloy is an ideal system which has moderate as well as controllable carbon solubility by tuning the atomic fraction of $\mathrm{Ni}$ in $\mathrm{Cu}$. To elucidate the behavior of these binary systems, Robinson et al.,[79] studied Cu-Ni foils (90/10\% in weight) from the catalyst point of view. Ni surfaces are more catalytically active than $\mathrm{Cu}$ surfaces so the rate of dissociation of the hydrocarbon precursor should be much higher on the alloy than on pure Cu surfaces. In principle, this could mean lower graphene growth temperatures. Nevertheless, relatively small, $100 \mu \mathrm{m}$, substrate grains were observed for the 90/10 $\mathrm{Cu}-\mathrm{Ni}$ foils annealed at 1050․ Therefore, either much longer annealing times or higher annealing temperatures are needed to grow centimeter sized substrate grains. In addition, an experimental study of the equilibrium surface composition of $\mathrm{Cu}-\mathrm{Ni}$ alloy substrates by Sakurai et al. [80] found strong $\mathrm{Ni}$ segregation for $\mathrm{Cu}$ rich alloys, which may enhance the catalytic activity further.

Growing experiments with different atomic fractions were also performed. A cold-wall chamber (8 Torr pressure and $100 \% \mathrm{CH}_{4}$ ) were used [78] to obtain monolayer, bilayer, and multilayer graphene as well as ultrathin graphite on commercial polycrystalline $\mathrm{Cu}-\mathrm{Ni}(31.00 \% \mathrm{Ni}, 67.80 \% \mathrm{Cu})$. In this case, the foil showed millimeter grains size after annealing. Different cooling rates ( 5 or $100 \stackrel{\circ}{\circ} / \mathrm{s})$ and temperatures $\left(930^{\circ} \mathrm{C}\right.$ to $\left.1030^{\circ} \mathrm{C}\right)$ were used. The observed uniformity of monolayer graphene on this $\mathrm{Cu}-\mathrm{Ni}$ alloy surface was much better than the reported results on polycrystalline Ni up to now.

Liu et al. [81] reported a facile segregation approach for this purpose using Cu-Ni mixtures with different percentages. Bulk nickel generally contains a trace amount of carbon species, which can be brought into the thin $\mathrm{Ni}$ film during electron beam deposition process. A sandwiched structure of $\mathrm{Cu}(370 \mathrm{~nm}) / \mathrm{Ni}(20-$ $130 \mathrm{~nm})(\mathrm{C}) / \mathrm{SiO}_{2} / \mathrm{Si}$, where the Ni layer was used as the carbon source (carbon content about $2.6 \mathrm{at} \%$ ) and the $\mathrm{Cu}$ layer was employed as a favorable segregation medium was grown. To date over $95 \%$ monolayer and $91 \%$ bilayer graphene films were prepared by only changing atomic percentage of $\mathrm{Ni}$ in $\mathrm{Cu}-\mathrm{Ni}$ alloy. For a Cu-Ni alloy having 5.5\% Ni component, monolayer graphene occupied over $95 \%$ of whole film. When $\mathrm{Ni}$ component in the alloy was increased to $10.4 \%$ the segregated film was dominated by bilayer graphene over $89 \%$. Further increasing the Ni percentage lead to thicker graphene.

\section{$\mathrm{Ni}-\mathrm{Au}$}

One more novel experiment was performed by Weatherup et al. [82]. This group was working in the design of alloy catalysts for low temperature synthesis of graphene. They showed that alloying polycrystalline $\mathrm{Ni}$ with $\mathrm{Au}$ ( $\mathrm{Ni}$ films $\sim 550 \mathrm{~nm}$ thick on $\mathrm{SiO}_{2}(300 \mathrm{~nm}) / \mathrm{Si}$ substrates covered with various thicknesses of thermally evaporated Au up to $10 \mathrm{~nm}$ ) allows MLG CVD at temperatures of 450ㄷ with reasonable crystallinity and domain sizes. This low-temperature would be compatible with back-end CMOS integration. AP, scalable chemical vapor deposition of predominantly monolayer (74\%) graphene films with an average D/G Raman peaks ratio of 0.24 and domain sizes in excess of $220 \mu \mathrm{m}^{2}$ was demonstrated. The Au alloying thereby drastically lowered the graphene nucleation density, allowing more uniform and controlled growth and highlighting the role of step edges. They also found that 3-5 $\mathrm{nm}$ $\mathrm{Au}$ gave the best graphene uniformity with regards to their CVD reference conditions with thicker Au layers leading to more inhomogeneous graphene layers. One drawback could be the preannealing that should be applied at higher temperatures to mix the alloy, although one advantage could be that mixing can be promoted by metal co-deposition. 


\section{Dielectric substrates}

Generally CVD graphene is transferred onto a desired substrate for further applications. Thus, it is of great interest to directly prepare continuous graphene films on dielectric substrates such as $\mathrm{BN}, \mathrm{Si}, \mathrm{SiO}_{2}, \mathrm{Al}_{2} \mathrm{O}_{3}$, $\mathrm{GaN}, \mathrm{MgO}, \mathrm{Si}_{3} \mathrm{~N}_{4}$ and so on. Considerable efforts were made to directly grow graphene films on these substrates, [83-88] but so far continuous and highly conductive films were very difficult to synthesize. Important applications were reported for the replacement of conventional transparent conductive films (TCFs), including $\mathrm{In}_{2} \mathrm{O}_{3}: \mathrm{Sn}$ (ITO), $\mathrm{SnO}_{2}: \mathrm{F}$ (FTO) and so on for graphene films. However, the highest efficiency achieved in solar cells was only 4.17\%, [89] which can still not compete with conventional solar cells.

There are some examples of experimental work on the synthesis of graphene over dielectric substrates. $\mathrm{Bi}$ et al. [90] reported the direct growth of graphene films on dielectric substrates by ambient pressure CVD (APCVD) at 1100-1200ㅇ $\mathrm{C}$ using a gas mixture of methane $\left(\mathrm{CH}_{4}\right)$, hydrogen $\left(\mathrm{H}_{2}\right)$ and argon (Ar). It is important to mention that at $1200^{\circ} \mathrm{C}$ over $\mathrm{BN}, \mathrm{Si}, \mathrm{SiO}_{2}$ and $\mathrm{AIN}$ substrates, not monolayer but few-layer graphene growth were reported.

Other singular approach was the direct chemical vapor deposition of a single or few-layer graphene film on dielectric surfaces via a sacrificial copper film [91] by Ismach et al. Working on the CVD growth of graphene on micrometer-thick copper foils they noticed that a significant amount of the copper evaporates and deposits at the edges of the fused silica tube used in the CVD.

Considering the melting temperature of the copper, $\sim 1084^{\circ} \mathrm{C}$, along with the high temperature during the growth, $\sim 1000^{\circ} \mathrm{C}$, and the low pressure in the chamber, 100-500 mTorr, the significant evaporation of the metal is not surprising. They proposed that if long processing time is used (up to $300 \mathrm{~min}$ ) the Cu film can evaporate completely. This CVD few-layer graphene exhibits the characteristics similar to that of turbostratic graphene, that is, lack of long order in the perpendicular direction. The results showed that the continuity of the metal film on the surface depends on its thickness, the metal-dielectric wetting properties, and the heating temperature and time. Typically, 100 to $450 \mathrm{~nm}$ thick $\mathrm{Cu}$ films on quartz and other insulating substrates were used. After the initial heating, the dewetting and evaporation rates of the metal decreased, presumably, due to the graphene growth that increases the coverage of the copper surface. Single to few-layer graphene film was obtained, but the film was highly defective and composed of thicker graphitic-like material and highly damaged graphene layers.

In conclusion, up to now many approach to control the number of layers via metal alloys catalysis were performed. There were some important advances on this subject with controlled few layers growth over alloys catalyst. Nevertheless, physical, optical and electronic properties of these films still have to be necessarily improved. Also it would be highly desirable to directly growth graphene layers on dielectric substrates. More experimental work should be performed in these areas for graphene to be competitive on practical applications.

\subsubsection{CRYSTALLOGRAPHIC STRUCTURE OF METAL SURFACE}

In the choosing of the catalyst, other parameters more than the material or chemical nature have to be taken into account. Crystal structure, texture, mono and polycrystalline character and the evolution of the surface morphology during synthesis, among others, were demonstrated to be critical aspects for graphene nucleation and growth.

\section{Single crystals, polycrystals and grain size}

There are examples of experiments where, even on a single metal grain (single crystal) different orientations of graphene domains were observed [92]. Moreover, in a recent work of Gao et al [71], they observed that a single-crystal Pt substrate showed similar growth behavior for graphene as that on 
polycrystalline Pt. Nevertheless, the size and orientation of the metal substrate grains were expected to have a large impact on the defect density of the graphene films grown by the dissociation of hydrocarbon molecules on metal substrates. The larger the grain size the higher-quality graphene films were demonstrated [7].

\section{Crystal structure orientation, texture and lattice match}

Graphene has a hexagonal-honeycomb lattice character in surface. Regardless of graphene can grow on several hexagonal or other crystallographic surfaces, growth on hexagonal substrates was frequently referred to as "epitaxial" even if significant lattice match was absent between the graphene and the substrate. Lattice mismatch of less than $1 \%$ is present on Co (0001) and $\mathrm{Ni}(111)$ surfaces. In contrast, lattice mismatch between graphene and $\mathrm{Cu}(111), \mathrm{Pt}(111), \operatorname{Pd}(111), \operatorname{Ru}(111)$ and $\operatorname{Ir}(111)$ is $>1 \%$ [14]. For systems with only a small lattice mismatch, a strong interaction can result in a pseudomorphic growth resulting in a large coincidence lattice and a "Moiré pattern" type of growth as a result of different rotational alignments [94].

In the case of copper, the role of $\mathrm{Cu}$ orientation and lattice mismatch was also more important than expected after the preliminary successful experimental results on polycrystalline $\mathrm{Cu}(100)$ foil surface [6]. A detailed study of the as-grown graphene showed that graphene had distinctive four-lobed islands and substantial in-plane rotational disorder [29]. The in-plane orientations were around two crystallographically equivalent $\mathrm{Cu}$ directions, a consequence of placing the sixfold graphene on the fourfold $\mathrm{Cu}(100)$ substrate. Furthermore, each nucleation site typically generated four graphene crystals, each with a different in-plane orientation. Island morphology was strongly determined by substrate temperature.

More recent works demonstrated that although Moiré patterns were observed on both $\mathrm{Cu}(111)$ [93] and $\mathrm{Cu}(100)$ [65], the hexagonal lattice of $\mathrm{Cu}(111)$ favored the high quality of as grown graphene. There were observed two predominant Moiré patterns in $\mathrm{Cu}(111)$ which could suggest that the graphene have preferred orientations with the underlying $\mathrm{Cu}(111)$. The results observed comparing the different orientations led to speculate that graphene could prefer nucleation on $\mathrm{Cu}(111)$ crystal plane than $\mathrm{Cu}(100)$ and (110), because of the similar crystallographic geometry in $\mathrm{Cu}(111)$ reducing so the nucleation barrier, since the lattice mismatch between graphene and underlying metal causes an additional energy cost.

Zhao et al. [94] also investigated the influence of the surface structure of single Cu crystals in UHV from ethylene at $900 \circ \mathrm{C}$. The over graphene had a hexagonal superstructure (Moiré) on $\mathrm{Cu}$ (111) and linear superstructure with a periodicity of $11 \mathrm{~A}$ and an angle of 0 o on $\mathrm{Cu}(100)$ square lattice. The graphene film properties were confirmed to be much poorer on the $\mathrm{Cu}(100)$ surface when compared to the $\mathrm{Cu}(111)$ surface.

Wood et al. [95], using different characterization techniques, found that substrate crystallography affects graphene growth even more than facet roughness. They determined that (111) containing facets produce pristine monolayer graphene with higher quality and growth rate than (100) containing facets, especially in $\mathrm{Cu}(100)$. This could be attributed to the high diffusion $[93,94]$ and improved adsorption of carboncontaining species on $\mathrm{Cu}(111)$ [96]. Since $\mathrm{Cu}(111)$ is the lowest-energy $\mathrm{Cu}$ surface,[97] longer pre-growth annealing treatments under $\mathrm{Ar} / \mathrm{H}_{2}$ flow at 900 ㅇ C could help for the production of $\mathrm{Cu}(111)$ facets on the polycrystalline $\mathrm{Cu}$ substrate. So, engineering $\mathrm{Cu}$ to have (111) surfaces is expected then to cause monolayer, uniform graphene growth.

Recently, "Orientation-controlled growth of graphene" was performed by an epitaxial CVD approach using heteroepitaxial $\mathrm{Co},[98] \mathrm{Ni},[99] \mathrm{Cu},[100,101] \mathrm{Ir},[102]$ and $\mathrm{Ru}[103,104]$ films deposited on single-crystal sapphire $\left(\alpha \mathrm{Al}_{2} \mathrm{O}_{3}(0001)\right)$ or $\mathrm{MgO}$ (111) substrates. This approach gave as a result crystalline metal films 
suitable for large-area graphene growth with much lower cost than single crystals, not only for low carbon solubility metal like Cu but also for Co and Ni whose carbon solubility is high. Ogawa el al.[105] compared domain structures of large-area, in single-layer graphene films grown on heteroepitaxial $\mathrm{Cu}(111)$ and (100) films both deposited by magnetron sputtering on single-crystal $\mathrm{MgO}(111)$ and (100) substrates, respectively. It was demonstrated that domain structure and size, as well as the orientation were strongly influenced by the $\mathrm{Cu}$ crystalline plane and that the $\mathrm{Cu}(111)$ was preferable for the orientation-controlled graphene growth. Graphene was epitaxially formed on $\mathrm{Cu}(111) / \mathrm{MgO}(111)$ but graphene/ $\mathrm{Cu}(100) / \mathrm{MgO}(100)$ showed a more complex LEED pattern where graphene covered the $\mathrm{Cu}$ surface with two preferential [10] orientations with angles of $0 \pm 2^{\circ}$ and $30 \pm 2^{\circ}$ with respect to the underlying $\mathrm{Cu}[011]$ lattice. LEEM measurements for the as-grown single-layer graphene on $\mathrm{Cu}(100)$ also showed that the as-grown graphene possessed a clear multidomain structure with patches of small domains.

In conclusion, it is worth to mention that even though in some experiments different orientations of graphene domains on a single metal grain were obtained, the hexagonal lattice of $\mathrm{Cu}(111)$ favored the high quality of as grown graphene. Moreover, during the CVD heteroepitaxial growth on $\mathrm{Cu}(111)$, the orientation of graphene nuclei became well controlled with domain boundaries atomically connected. This could represent a new alternative. Therefore, it could be advantageous to develop techniques for producing foils of these materials with a (111) surface texture.

\subsubsection{THICKNESS, ROUGHNESS AND MORPHOLOGY}

\section{Thin films and Foils}

Standard processes in Si technology include, among others, the deposition of $\mathrm{Cu}$ and $\mathrm{Ni}$ thin films on $\mathrm{Si}$ substrates and wafers. The evaporation of metals is performed in HV systems and is not a cheap process to manage in lab research. Therefore, a lot of lab research in graphene synthesis was performed using different catalyst configurations as thin foils (up to 25-30 $\mu \mathrm{m}$ thick). These foils were cheaper than films deposition processes and all the processes developed on foils can be translated easily to thin films and their standard processes. Another advantage is that foils are flexible and even though their initial high roughness, it is also well known that annealing the foil metal can lead to very large grain size, with a flattened surface that rivals or even exceeds the flat surfaces typically obtained by cutting and polishing single crystals. After a typical annealing process, the spaces between single atomic steps in the case of $\mathrm{Cu}$ are often greater than $100 \mathrm{~nm}$, indicating surface normal less than $0.1^{\circ}$ from the [100] azimuth. [29].

It is worthy of mention that the lateral dimensions of the $\mathrm{Cu}$ grain boundaries were observed to vary with the annealing pre-treatment time and also with the $\mathrm{Cu}$ foil thickness[14]. $\mathrm{H}_{2}$ embrittlement of $\mathrm{Cu}$ could also limit diffusion and minimizes grain growth during annealing.

In this connection, the pioneering work of large area graphene growth on Cu foils $[6,31,59]$ developed in low pressure (LP) CVD at $1000{ }^{\circ} \mathrm{C}$ from $\mathrm{CH}_{4}$ and $\mathrm{H}_{2}$ mixtures on foils of various thickness $(12.5,25,50 \mu \mathrm{m})$ to check precipitation mechanism, did not show evidence of differences in graphene quality. Moreover, the cheap copper foils revealed ideal for large area graphene synthesis, up to 30 inch demonstrated [7]. On the other hand, it is commonly accepted that commercial $\mathrm{Cu}$ foils are (100) textured after annealing (and due to the cool rolling fabrication process) and as seen above, the crystallography strongly affects the graphene growth. Pregrowth low-energy electron diffraction (LEED) analysis of the Cu surface also showed that annealing resulted in a (100) texture in the foil plane [29].

Returning again to standard processes, in high performance electronic devices, the graphene must be electrically isolated from its surrounding environment. In the case of copper, this is generally achieved by etching the underlying metal substrate, with subsequent transfer to an insulating substrate, such as silicon oxide [6]. However, such a layer transfer process is non-ideal for nanoelectronic applications due 
to microcracking and the potential for interfacial contamination between the graphene and the substrate. Levendorf et al [106] demonstrated a transfer-free process for fabrication of CVD graphene transistors. Instead of utilizing a bulk copper substrate, an evaporated copper film on an oxidized silicon wafer was grown. One problem with the evaporated $\mathrm{Cu} / \mathrm{SiO}_{2} / \mathrm{Si}$ system is that, although stable at temperatures $<800 \circ \mathrm{C}$, it was unstable at the temperature used on the synthesis. One approach to ensuring stability at the $\mathrm{Cu} / \mathrm{SiO}_{2}$ interface could be to minimize the graphene synthesis temperature. Another approach presented evidence of significant $\mathrm{Cu}-\mathrm{Si}$ interdiffusion during graphene synthesis and evaluated the use of metal and insulating diffusion barriers as a means of prevention at the $\mathrm{Cu} / \mathrm{SiO}_{2}$ interface [107]. Diffusion barriers $\left(\mathrm{W}, \mathrm{Cr}, \mathrm{Ni}, \mathrm{Al}_{2} \mathrm{O}_{3}, \mathrm{HfO}_{2}, \mathrm{SiN}_{\mathrm{x}}\right.$ ) were demonstrated to reduce interfacial diffusion, but often the $\mathrm{Cu} / \mathrm{Si}$ interdiffusion was not completely suppressed. To solve this, instead of using a similar process to grow on freestanding foils, the pre-growth annealing of the foil at a lower T (typically $700 \circ \mathrm{C}$ ) was carried out to achieve $\mathrm{Cu}$ grain growth, that also minimized the copper evaporation occurring at high temperature. Moreover, excessive exposure to high temperatures can result in the formation of voids in the copper film and decomposition of the film into islands.

Another reason for using thin films, mostly in the case of $\mathrm{Ni}$ and other metals with high solubility, was that the large amount of carbon sources absorbed on nickel foils usually formed thick graphite crystals rather than graphene films $[66,108]$. To solve this problem, very thin layers of nickel of thickness down to 300 $\mathrm{nm}$ were deposited on $\mathrm{SiO}_{2} / \mathrm{Si}$ substrates using electron-beam evaporators. It was demonstrated that very thin films and rapid cooling proved critical in suppressing formation of multiple layers.

\section{Macro-roughness and morphology}

It was often found that the topography of the surface strongly affects the uniformity of grown graphene. Also it was widely discussed that the purity of the film determines the number of layers, mainly at low pressure conditions [30]. Luo et al., [109] published that the use of very flat, electropolished Cu catalyst surface and extremely low methane concentration enables the growth of a very uniform graphene film. Boundary structures of the standard $\mathrm{Cu}$ foil led to thickness variations in the graphene film. Raman measurements of the graphene film regions that replicate the $\mathrm{Cu}$ grain boundary regions revealed carbon atoms in disordered $\mathrm{sp}^{3}$-bonded networks in these areas. The correlation of graphene thickness variation with the topography of the catalytic $\mathrm{Cu}$ foil motivated the idea that smoothing the $\mathrm{Cu}$ through polishing would lead to more uniform and better quality graphene. Moreover, first principles calculations indicated that, in contrast to graphene growth on other metals, Cu-catalyzed graphene nucleation was particularly favoured at surface irregularities (i.e., metal step edges and other defects) but also occurs over the flat regions [110]. Therefore, nucleation was also found to proceed readily on the crystal plane and to be favoured at high partial pressures.

\section{Micro-roughness, boundaries, step edges}

It was published in theoretical works that carbon binds stronger at the step edges than on the terraces for transition metals as $\mathrm{Ni}, \mathrm{Co}, \mathrm{Ru}$, and $\mathrm{Rh}[111]$. Even with full coverage along the step edge, carbon still binds most favorably to the step sites $[112,113,114]$. Metal atomic step edges were also demonstrated to be important for graphene island nucleation in the case of $\mathrm{Cu}$, but not unique and in some cases not the most desired.

Regarding this, Wofford et al., [29] published that the minimum temperature of $\sim 790{ }^{\circ} \mathrm{C}$ is sufficiently high to induce significant motion of $\mathrm{Cu}$ steps due to sublimation on $\mathrm{Cu}(100)$ foils in LP CVD. Real time observations during synthesis demonstrated at high temperatures above $9600^{\circ} \mathrm{C}$, that the surface of each $\mathrm{Cu}$ grain consists of a propagating array of monolayer-height steps before growth. Subsequently, when a segment of a $\mathrm{Cu}$ step edge collides with a growing graphene island, it decelerates and incoming step 
edges become bunched under the graphene. As a consequence incoming steps wrap around under the interior of the four-lobed graphene island. This process creates four-lobed $\mathrm{Cu}$ hillocks draped by graphene. Indeed, at very high temperatures surface roughening can be so dramatic that individual hillocks can be easily visible with an optical microscope. While single Cu step edges was not observed to have a perceptible effect on the growth, large bunches were observed to distort island evolution, to induce rotational disorder by altering growth trajectories and to decrease the graphene quality.

In the case of graphene islands grown on $\mathrm{Cu}(111)$ in UHV conditions it was also observed that $\mathrm{Cu}$ (111) step bunches led to rotational disorder in two ways [115]. First, they can cause islands to be nucleated with different in-plane orientations. Second, step bunches can generate new rotational boundaries as islands expand. Change of island orientation was observed when crossing a step bunch. Thus, fewer step bunches led to fewer rotational boundaries, consistent with the work of Zhao et al. [94] These high angle rotational boundaries could be minimized using higher growth temperatures in this $\mathrm{Cu}(111)$ case.

The tendency to introduce rotational boundaries during growth differentiates $\mathrm{Cu}(111)$ and (100) from other surfaces. For example, graphene sheets were observed to grow without changing orientation across boundaries between rotationally misoriented $\mathrm{Ru}(0001)$ grains [116] and even across different facets of $\mathrm{Ni}$ grains. It is worthy of mention that temperature and pressure dependence of this processes have to be taken into account. As we discuss below, compact hexagonal shapes were observed in high-pressure CVD by several groups. The carrier and carbon-source gases in CVD suppress $\mathrm{Cu}$ evaporation so that a higher temperature can be employed compared to that used with UHV growth. At high temperatures in UHV experiments, the surface morphology is evolving quickly due to sublimation, causing large step bunches to collect at graphene edges. Rearranging these step bunches is likely difficult, impeding the processes that lead to hexagonal shapes. Therefore, pressure and temperature seem to have a critical role in evaporation and propagation of metal atomic steps.

\section{Other defects and impurities}

It was observed that graphene initially nucleates on the $\mathrm{Cu}$ surface impurities, imperfections, step edges and grain boundaries. So, reducing the density of nucleation sites can effectively increase the graphene domain size [65]. It was also observed that the type of nucleation site can affect whether the graphene island was single crystal or not [29] that gives rise to a mean grain number much smaller than the nucleation density. Polycrystals are preferentially formed on defective nucleation sites whereas single crystals do preferentially at the less defective regions. On $\mathrm{Cu}$ (111), single crystal graphene firstly nucleated inhomogeneously at defects such as steps, step bunches, and impurities [115] but changing to proper synthesis conditions can lead to secondary single crystal nucleation, more difficult to achieve because of the more difficult nucleation on less defective regions. The metal purity was considered as a meaningful aspect to yield higher quality graphene. It was published that higher purity copper foils (99.999\%) yield higher quality graphene with room-temperature mobility closer to those reported for exfoliated graphene [117]. Kalbac et al. [28] suggested that the graphene growth begins by the formation of a multilayer cluster due to complex defects or polycrystalline impurities. This seed was observed to increase its size but the growth speed of a particular layer depends on its proximity to the copper surface.

Liu et al., [30] found that the purity of the Cu film clearly determined the number of synthesized graphene layers at low pressure conditions. Multilayer graphene was observed to nucleate from the impurity clusters and merge into a 1-2 layer graphene film away from the nucleation centers.

The above discussion reveals that it is crucial to reduce the number of imperfections, roughness and impurities and make the surface as uniform as possible to obtain high-uniformity graphene on $\mathrm{Cu}$. It is clear that the roughness and imperfections initially enhance graphene nucleation, but also defective graphene later. It is also possible that the impurity atoms in the bulk diffuse to the $\mathrm{Cu}$ surface during annealing. These surface impurities dramatically enhance the catalytic capability of $\mathrm{Cu}$ resulting in high 
concentration of carbon atoms that are decomposed from precursors. Hence, it can be concluded that the purity of $\mathrm{Cu}$ surface plays a critical role in determining the number of graphene layers.

\subsubsection{SEEDED GROWTH AND CONTROLLED NUCLEATION}

Recently, new approaches dealing with pre-patterned graphene seeds were investigated. Substrate surface seeding was a common surface pretreatment method used to modify and control the surface nucleation density and the growth rate of diamond films [118]. In the case of diamond, powder or particles littered on the substrate surface, were used as the predominant nucleation sites and the material gradually was grown owing to the $\mathrm{C}$ ad atom concentration.

In the case of graphene on $\mathrm{Cu}$, these new experiments were performed in different ways, either using seeds of prepatterned multilayer graphene flakes or controlling nucleation by locally providing a high concentration of carbon. In the former method [119] however, an extra CVD process was firstly required to obtain a continuous multilayer graphene film on $\mathrm{Cu}$ used for the following lithographic patterning of the growth seeds (multilayer graphene). In the latter method [120], polymethylmethacrylate (PMMA) was used as a carbon source for enhancing local nucleation. This latter method demonstrated controlled graphene nucleation and synthesis of single crystal graphene arrays and offered a promising route to fabricate graphene-based devices free of grain boundaries and with more reliable performances. Atmospheric pressure CVD (APCVD) was used in this method. Occasionally, polycrystalline islands or fewlayer domains, nucleated and grown from one single such nucleus, were also observed.

Some experiments with highly oriented pyrolytic graphite (HOPG) flakes as seeds transferred to the Cu foil surface, simply by pressing against the patterned HOPG were also performed. After the CVD synthesis, a preferential nucleation of graphene on HOPG sites was also observed. However, due to the roughness and flexibility of the surfaces of those thin $\mathrm{Cu}$ foils, transferred graphite flakes differ significantly in size, thickness and shape affecting graphene growth. In addition, it was nearly impossible to fabricate welldefined arrays by the pressing method.

This seeded growth could be a very interesting alternative for high quality and performance graphene layers with applications in several fields. Nevertheless, very high complex processes with important economical implications are involved.

\subsection{THERMODYNAMIC AND KINETIC PARAMETERS}

Pressure and temperature revealed as key factors during graphene growth. Other dynamic factors, such as gas flow and gas residence time inside the processing chamber, also play important roles that will be reviewed in this section. We will also refer to other important aspects of the synthesis such as plasma assisted deposition and different time profiles during all the steps of the graphene deposition.

\subsubsection{PRESSURE}

Vacuum level prior synthesis should be minimized to the limit in order to get high purity layers. The lower the base pressure of the reactor, the lower density of impurities and residual oxygen may remain. Working in low pressure CVD, a few mTorr vacuum level has been commonly used. On the other hand, recipes with diluents $\left(\mathrm{H}_{2}\right)$ of the precursor gases have been proposed for reducing residual oxygen during annealing and growing stages of the synthesis.

Up to now, a lot of experimental work was published in a extensive pressure range, $\mathrm{HV}$ (High Vacuum, $10^{-}$ ${ }^{4}-10^{-6}$ Torr), LP (Low Pressure 0.1-1 Torr) and AP (Atmospheric Pressure). It was possible to grow-graphene with acceptable quality in all ranges, but great differences in graphene domain size and morphology were 
observed. In LP CVD flower like-four to six lobed structures were commonly grown $[6,59]$. In contrast, in AP CVD, hexagonal structures of graphene were synthesized [121]. Concerning the domain size, both cases offer graphene flakes near milimeter size and continuous monolayer films when using Cu catalyst. Bhaviripudi et al., [121] studied, under the same experimental setup, the role of the total pressure on Cu. The results revealed that even though the thermodynamics of the system remains the same, whether the process was performed at AP,LP or under UHV conditions, the kinetics of the growth phenomenon was different.

Although in LP, monolayer graphene was grown (up to 95\% monolayer coverage), in the case of AP growth, results varied from a monolayer at lower methane concentrations (parts per million concentration) to multilayer domains with a monolayer graphene background at higher methane concentrations $(5-10 \%$ by volume). This seems to indicate that either the growth was not self-limiting under higher methane concentrations or only partial coverage was achieved. This lack of control over the number of layers and the different domain shapes promoted further investigations performed to determine the detailed growth mechanism.

The more exciting difference between LP and AP maybe, the morphology and shape of the graphene domains, already were extensively studied in real time LP experiments $[29,115]$. In the case of AP the tendency for the graphene grown into AB Bernal few-layer hexagonal stacks was also studied [122]. It was discussed that the growth of top graphene layers with no contact with the copper surface could be due to the high local supersaturation of carbon at high methane flow rates with an excess of precursor supply. Higher precursor supersaturation should lead to considerably faster graphene growth. However, low graphene coverage rate of $0.2 \mu \mathrm{m}^{2}$ per minute was monitored, whereas considerably higher rates of up to $100 \mu \mathrm{m}^{2}$ per minute were observed for low pressure growth by Li et al. [31] It could be hypothesize that the lower growth rate could be due to lower lateral diffusion rate of species that favour multilayered graphene instead of monolayer fast growth.

To better support the discussion of the formation of multi-layer graphene at an early stage and the limited further growth of the top layers Wu et al., [123] developed a two-step ambient pressure CVD process with similar strategy as Li et al. The result was consistent with the point that, once a continuous graphene film formed on the catalytic $\mathrm{Cu}$ surface, growth of extra graphene layer was inhibited because of the absence of $\mathrm{Cu}$ to catalytically decompose the carbon precursor gas [27].

Another significant factor related to the experimental pressure is the sublimation-evaporation during the process due to the vapor pressure $\left(V_{p}\right)$. The vapor pressure of the substrate was demonstrated to have a strong influence on the growth rate and orientation of the graphene grains [29] [79]. $\mathrm{V}_{\mathrm{p}}$ of $\mathrm{Cu}$ and $\mathrm{Ni}$ are $6 \cdot 10^{-5}$ and $1 \cdot 10^{-7}$ Torr, respectively. For the $\mathrm{Cu}(111)$ surface, this represents a loss of 4 monolayer $(\mathrm{ML})$ of $\mathrm{Cu}$ per second from the surface at $1000{ }^{\circ} \mathrm{C}$ under ultrahigh vacuum conditions, whereas the sublimation rate from a $\mathrm{Ni}(111)$ surface is approximately $7 \times 10^{-3} \mathrm{ML} / \mathrm{s}$. For graphene growth on $\mathrm{Cu}$ substrates in LP regime the pressure of the source gas is typically in the mTorr or higher pressure range, which should slow the sublimation rate of $\mathrm{Cu}$ from the surface. Even so, sublimation of metal during growth was reported due to high temperature commonly used, close to the melting point of the copper, 1084 으, and even more in LP regime. Significant amount of the copper evaporates and deposits at the edges of the fused silica tube used in the CVD. However, once graphene growth was initiated, the graphene covered regions of the surface were observed to suppress sublimation.

Sublimation of the metal catalyst during the growth was used to deposit monolayer graphene, although this parameter should be precisely controlled, [91] [124]. Defective films were grown using sacrificial copper films. One cause of the defective film could be that the grown graphene can break under stress due to the Cu morphology change during its evaporation. 
It is worth mentioning that Liu et al., [30] noticed that decreasing the annealing pressure (from 80 to 20 mbar) could have a positive effect on the quality and uniformity of graphene. It was observed that the surface of $\mathrm{Cu}$ became smoother due to the increasing sublimation of $\mathrm{Cu}$ at lower pressure. Low pressure during annealing could greatly enhance the uniformity of $\mathrm{Cu}$ surface and decrease the number of the sharp structures, thereby making the $\mathrm{Cu}$ surface smoother. But it was already demonstrated that in the growing stage [29] Cu sublimation should be avoided due to roughening of the Cu surface and, thus, the conformal graphene film.

\section{Partial pressure of precursor gases and hydrogen}

Recent studies on the graphene growth optimization $[31,123]$ indicated that hydrocarbon pressure is one of the major factors affecting the graphene growth. A minimum partial pressure of hydrocarbon is required in most of the experiments for graphene to cover the Cu surface during growth. The pressure of the hydrocarbon determines the concentration of the carbon species on the $\mathrm{Cu}$ surface during graphene growth. So, a controlled and relatively low precursor $\left(\mathrm{CH}_{\mathrm{x}}\right)$ partial pressure is highly recommended in all pressure ranges $[109,121]$. It was also demonstrated by Li et al., the synthesis of high quality graphene working at very low total pressure (mTorr range) with almost only carbon precursors and low quantity of $\mathrm{H}_{2}$.

Zhang et al., [125] investigated the correlation between the grain morphology and the total pressure and methane to hydrogen ratio in LP. With methane-to-hydrogen ratio of $1: 12.5$ and at different total pressures, the graphene grains changed from irregular small flakes ( $80 \mathrm{mTorr}$ ) to mostly four-lobe grains(100 mTorr) and finally to mostly six-lobe flowers (150 and $200 \mathrm{mTorr}$ ). When increasing the total pressure to 300 mTorr, the six-lobe graphene flowers turned to irregular shape. Interestingly, similar results were obtained when keeping the total pressure at 150 mTorr and gradually increasing the methane to hydrogen ratio from 1:30 to 1:2. All these observations indicated that increasing the total pressure of the CVD system had a similar effect on the morphology of graphene grains as increasing the methane-to-hydrogen ratio.

The role of hydrogen partial pressure was already commented (see section 3.1). Gao et al., [71] observed that the graphene edges become regular and straighter, because edges with a low stability were selectively etched away by an active atomic $\mathrm{H}$ when the $\mathrm{CH}_{4} / \mathrm{H}_{2}$ ratio is low. Wang et al., [126] also published about the role of hydrogen. They observed that when only the $\mathrm{CH}_{4}$ supply was shut off and the sample was cooled, after the growth stage, in a high concentration $\mathrm{H}_{2}$ atmosphere $(300 \mathrm{sccm} \mathrm{Ar}$ and 40 sccm $\mathrm{H}_{2}$ ), the graphene domains would appear to be etched into rectangular openings. In accordance with the copper-catalyzed etching mechanism proposed by Zhang et al. recently, [127] they proposed that low concentration $\mathrm{H}_{2}$ could reduce the etch rate considerably and result in no significant etching damage.

The whole section reveals that the pressure regime is crucial in high quality graphene synthesis. It seems that more regular graphene domains are synthesized in AP CVD. LP CVD regimes enhance evaporation of catalyst and etched domains, but more precise control of the number of layers is achieved. The role of hydrogen remains an open question to be resolved, although etching effects were demonstrated. The partial pressure of the precursor gases reveals as crucial and dependent of each reactor setup although some relation between this partial pressure and the total pressure of the system was detected.

\subsubsection{TEMPERATURE}

One important effect, widely detected in tubular reactors is the temperature gradient along the radial direction inside the reactor. It can result in inhomogeneous growth of the graphene. To solve this, two 
quartz tubes, one suspended inside the other, were used in large area synthesis experiments, wrapping the small tube with a metal foil [7].

\section{Heating treatment of the catalyst}

In catalysis, it was demonstrated an enhanced dissociation of carbon precursors at high temperature and also surface smoothing via metal grain growth, being this a fundamental step in graphene synthesis over metal foils. Depending on the catalytic activity of the metal, the process temperatures can vary between $800-1100$ - $\mathrm{C}$. $\mathrm{Ni}$ is intensively studied because the phase diagram of $\mathrm{Ni}$ and $\mathrm{C}$ reveals that at high temperature solid solution is formed (above $800 \mathrm{\circ} C$ ) and that the metastable formation of $\mathrm{Ni}_{3} \mathrm{C}$ phase promotes the precipitation of carbon out of $\mathrm{Ni}$. Co and Fe show also carbon solubility at $850 \circ \mathrm{C}-1000$ 으 although graphite precipitation from $\mathrm{Fe}$ can be obtained only under very specific conditions. Working with $\mathrm{Cu}$ and $\mathrm{Ni}$ is common to use $900-1050 \circ \mathrm{C}$ temperature. It was demonstrated that high $\mathrm{T}(>1035 \circ \mathrm{O}$ ) yields a low density of graphene nuclei when growing and, as a consequence, large domain size. But the problems with high $\mathrm{T}\left(\mathrm{o}^{\mathrm{C}}\right)$ were that more metal was observed to evaporate depending on pressure conditions and that the roughness of the surface was promoted.

The "hot-wall" reactor consisting in a quartz tube located inside a furnace is the most used configuration in graphene synthesis so far $[7,129,130]$. On the other hand, it is believed that, in this type of reactors, the thermal gas activation and dissociation in gas phase may be considered as not specially noticeable. Therefore, if the reaction is surface limited (heterogeneous reaction) there is no need to heat the gas up to this high temperature before reaching the surface. There is another possibility, a design called "coldwall type" apparatus where only the substrate is heated and in less time. The cold wall type reduces completely gas phase reactions resulting in no particulate contamination. Synthesis of high quality graphene films on a $\mathrm{Ni}$ foils using a cold-wall reactor with a rapid thermal processing (RTP) heater was reported [128]. RTP provided a fast heating \& cooling rate and temperature control. The reported results differed from previous observations in the growth of graphene on Ni substrates by CVD due to the rapid process, even allowing new discussions about growth kinetics. These results suggested that two different growth mechanisms could exist in the case of $\mathrm{Ni}$. While portion of carbon atoms may be dissolved into the nickel film, many carbon atoms also migrate on the nickel surface and bond with each other to form graphene. Therefore, this could explain the graphene synthesis in a very short growth time $(30 \mathrm{~s})$; this direct growth-surface migration mechanism may play a larger role than the precipitation mechanism. To verify the direct growth mechanism of CVD process very short growth time (10 s) and different $\mathrm{H}_{2}$ gas flow rates during the growth stage were investigated. The defect density decreased with decreasing the $\mathrm{H}_{2}$ flow rate. It could be postulated that hydrogen is not necessary to synthesize high-quality graphene by RTP direct growth mechanism using a Ni catalyst, consistent with the previously observed in the case of CVD growth of graphene on Cu foils [131].

Geng et al., [132] demonstrated in AP that the use of liquid $\mathrm{Cu}$ ( $T>1084{ }^{\circ} \mathrm{C}$, $\mathrm{Cu}$ melting point) can be particularly effective for controlling the nucleation process in graphene CVD systems. It was observed that the grain boundaries were eliminated and the results showed the production of uniform, self-aligned, large-sized, single-domain, hexagonal graphene flakes (HGF) and continuous monolayer films. This new route involved the formation of liquid Cu phase on quartz and W substrates at growth temperature above Cu melting point. The evolution from well-separated HGFs, to closely packed structures and to continuous film was documented. Typically, HGFs well dispersed on the surface and self-assembled were observed. Liquid $\mathrm{Cu}$ surface could be involved in the translation or rotation of HGFs, while minimization of surface energy may be responsible for the alignment. The average size of individual HGFs determined by both nucleation density and growth rate was about 20-30 $\mu \mathrm{m}$. Increasing growth temperature led to HGFs with average sizes of approximately $50 \mu \mathrm{m}$; and lowering $\mathrm{CH}_{4}$ flow rate led to approximately $120 \mu \mathrm{m}$. The average growth rate of HGFs was estimated to be $10-50 \mu \mathrm{m} / \mathrm{min}$ on flat $\mathrm{Cu} / \mathrm{W}$, which is much higher than the rate of $0.1-0.2 \mu \mathrm{m} / \mathrm{min}$ observed for the case of HGFs grown on a Cu solid surface [133]. 


\section{Low temperature synthesis (Plasma assisted processes)}

Low temperature processes for graphene synthesis are highly recommended for industrial applications. There is still a need to develop a reliable and reproducible method for low temperature synthesis of highquality graphene for the full exploitation of graphene properties and application potentials. One gamechanging breakthrough [134] would be the development of graphene growth on arbitrary surfaces at low temperatures (for example, by means of plasma assisted deposition) with a minimal number of defects. So, plasma assisted deposition could be a fundamental player in graphene future development.

Different works using surface wave plasma (SWP)-CVD and remote plasma assisted CVD [135] exhibited a capacity to synthesis graphene at lower temperatures and over substrates other than used in standard processes (Al foil). In the former method no monolayer graphene was deposited but few layer, graphene was synthesized at a relatively higher temperature of 650 ㅇ $\mathrm{C}$ in the latter method. In another approach by Kim et al., [136], graphene films were synthesized on polycrystalline nickel foil using a cold-wall type microwave MPCVD system with a heating stage. A substrate temperature of 450 to $750 \circ \mathrm{C}$ and a total pressure of 20 Torr under various mixing ratios of $\mathrm{H}_{2}$ and $\mathrm{CH}_{4}$ were used. The dependence of monolayer graphene synthesis on temperature was investigated and it was clearly shown that the higher the temperature the higher quality graphene was grown so far.

Teresawa et al., [137] investigated multilayer graphene grown on $\mathrm{Cu}$ foils by radio frequency plasma enhanced (RF) chemical vapor deposition (PE-CVD). The growth of graphene was investigated at various conditions, changing the plasma power, gas pressures, and the substrate temperature (from $500{ }^{\circ} \mathrm{C}$ to $\left.900^{\circ} \mathrm{C}\right)$. At high substrate temperatures, the growth of the first layer of graphene was affected by the catalytic action of $\mathrm{Cu}$, while the growth, at low temperature, of multilayer graphene was dominated mostly by radicals generated in the plasma. Outstanding differences in the grain size, number of layers and growth rates on few layer graphene between 500 and $900^{\circ} \mathrm{C}$ respectively were observed. It was also observed that the grain size of graphene decreases with the thickness. The growth rate of the subsequent layers in multilayer graphene was measured approximately five times slower than that of the first layer graphene. In thermal CVD, the difference in the growth rate between the first layer and the second layer was more than 10 [27]. This more rapid growth comparing with thermal CVD is one of the features in PECVD. In contrast to thermal CVD, the activated carbon fragment such as C2 radical is formed in PE-CVD

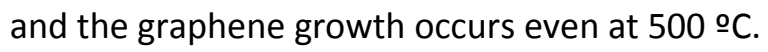

Rapid synthesis radio-frequency plasma-enhanced chemical vapor deposition (RF-PECVD) [138] revealed as a very powerful technique for the synthesis of large-scale graphene at relatively low temperature in a short time. Large-area single- or multilayer graphene of high quality was synthesized on Ni films deposited on a thermally oxidized $\mathrm{Si}$, at a relatively low temperature $\left(650^{\circ} \mathrm{C}\right)$. In the deposition process, trace amount of $\mathrm{CH}_{4}$ was introduced into the PECVD chamber and only a short deposition time (30-60 s) was used. Single or multilayer graphene were obtained due to carbon atoms from the discharge diffuse into the $\mathrm{Ni}$ film and then segregate out at its surface. Increasing number of graphene layers were obtained with longer deposition times using larger $\mathrm{CH}_{4}$ flows at a cooling rate of about $10^{\circ} \mathrm{C} / \mathrm{s}$. Kalita et al., [139] reported direct synthesis of nanographene films (very small domain size) on silicon ( $\mathrm{n}-\mathrm{Si})$ and glass $\left(\mathrm{SiO}_{2}\right)$ substrates by microwave assisted surface wave plasma (MW-SWP) CVD at $400-560$ ㅇ․ The technique is a rapid growth process (70-120 s) and the film can be deposited on different substrates. The directly grown deposit consisted of triangular shaped nanographene domains with sizes of $80-100 \mathrm{~nm}$ in lengths that interconnect to form a continuous film. For the deposition process, a gas mixture of $\mathrm{C}_{2} \mathrm{H}_{2}$ and $\mathrm{Ar}$ at a pressure of $45 \mathrm{~Pa}$ was used. Kumar et al., [140] reported a unique process for rapid synthesis $(100 \mathrm{~s}$ duration) of few-layer graphene films on $\mathrm{Cu}$ foil by microwave plasma chemical vapor deposition (MPCVD). The process can produce films of controllable quality from amorphous to highly crystalline by adjusting plasma conditions during growth and with no supplemental substrate heating (plasma-metal 
coupling for rapid heating of the foil). The hydrogen plasma was also used to remove the native oxide layer enabling graphene growth on metal $\mathrm{Cu}$. It was suggested that the same process could be used for rapid synthesis of primarily single-layer graphene.

Great advances are being published in this area of graphene synthesis. Monolayer high quality graphene on metal was already synthesized at lower temperatures. Synthesis of small domain graphene on dielectric substrates was also published. Therefore, low temperature processes for graphene synthesis are being developed by means of plasma assisted deposition. This will be an extensive area of research in the near future with the intention to synthesize controllable number of graphene layers on arbitrary substrates with a reliable and reproducible method.

\subsubsection{FLOW RATE AND RESIDENCE TIME}

Gas flow regime revealed as an important factor to be taken into account during graphene synthesis. It was also observed [31] that the density of graphene nuclei decreased as $\mathrm{T}(\mathrm{O} \mathrm{C})$ increased or as methane flow JMe $(\mathrm{sccm})$ and methane partial pressure PMe (mTorr) decreased. Therefore, high T and low JMe and PMe were found to yield a low density of graphene nuclei and thus large domain size. Different vapor trapping methods have been reported. Li et al. [59] demonstrated the CVD growth of graphene single crystals up to $0.5 \mathrm{~mm}$ in size in quasi-static flow regime (vapor trapping method), using a copper enclosure in LP. Subsequent experiments [125] in quasistatic flow achieved the growth of large-grain, single-crystalline six-lobe graphene flowers with grain size up to $100 \mu \mathrm{m}$. SEM images of these six-lobe graphene flowers grown on the bottom side of $\mathrm{Cu}$ foil placed inside a vapor trapping tube were shown. Interestingly, the graphene that grown on a Cu foil placed outside the small vapor trapping tube did not show any "flower" shape, but continuous graphene film with slight etching. This result indicated that the vapor trapping tube changed the local environment inside the tube, especially in reducing the carbon supply and creating a quasi-static reactant gas distribution that resulted in large flower shaped graphene grains.

Following this line of experimentation, when methane flow rate decreased (from $300 \mathrm{sccm}$ to $180 \mathrm{sccm}$ ) using Fe foils while keeping the other parameters unchanged [76] bilayer graphene instead of multilayer was obtained, likely due to the smaller amount of carbon dissolved and segregated from the catalyst.

\subsubsection{HEATING/COOLING TREATMENTS AND PROCESS IN STEPS}

\section{Annealing time}

Reducing the nucleation density was a feasible route to prepare large-size single-crystal graphene domains [59]. The presence of impurities and defects on the surface of a substrate proved to affect the nucleation behavior considerably. Regarding the importance of surface defects, Gao et al. [143] demonstrated that the graphene nucleation rate near a step edge may be $10^{4}-10^{7}$ times greater than that on a terrace due to a significantly lower nucleation barrier. As known, high temperature treatments of the substrate revealed to be helpful for the reduction of volatile impurities, contaminants, and defects on a copper surface, thus leading to the hindering of graphene nucleation. Very longtime annealing $(3 \mathrm{~h})$ was also used by Wang et al., [126] to reduce the nucleation density on copper foils resulting in large hexagonal domains about $0.4 \times 0.4 \mathrm{~mm}^{2}$.

The effects of total annealing time and temperature on the orientation and size of grains within $\mathrm{Cu}$ foils and $\mathrm{Cu}-\mathrm{Ni}$ alloy foils were also studied [79]. As above discussed, alloying metals is often performed in order to control the number of graphene layers. The annealing process could be summarized in two stages. During the grain growth, the first step is to incorporate and order atoms from the disordered regions, resulting in some ordered regions. The second step is the growth of large grains at the expense of 
smaller ones. As the temperature increases to a value close to the melting point of the metal, the diffusion coefficient of the atoms within the foil becomes very large (atomic diffusion is a thermally activated process), resulting in the growth of grains with macroscopic dimensions. The obtained results showed typical lateral dimensions ranging from a few millimeters up to approximately a centimeter for $\mathrm{Cu}$ foils annealed at $1030^{\circ} \mathrm{C}$ for $35 \mathrm{~min}$ and from tens of microns up to a few hundred microns for the $90 / 10$ $\mathrm{Cu}-\mathrm{Ni}$ foils annealed at 1050 으 for times ranging from 45 to $90 \mathrm{~min}$. The smaller grains within the $\mathrm{Cu}-\mathrm{Ni}$ foils were attributed to the higher melting point of the $\mathrm{Cu}-\mathrm{Ni}$ alloy. Then at these temperatures, more density of grain borders and subsequent defects on as-grown graphene can be possible to occur on alloys.

Concerning the effect of $\mathrm{H}_{2}$ during the annealing treatment, it is believed that $\mathrm{H}_{2}$ can eliminate certain impurities such as sulphur (S) and phosphorus (P) that may cause local variations in the carbon dissolvability in the metal substrates [144].

Therefore, it seems clear that long annealing times at low pressure of reducing gases combined with high temperature, yielded low nucleation density and subsequent large domains. This route is highly desirable in high quality graphene synthesis.

\section{Growing step profile}

In the pioneering work of Li et al., [31] a two step process in LP CVD, varying the partial pressure of methane for large domain graphene synthesis, has been proposed. For a given temperature (usually 1035 oC) nuclei were formed in a first step at low methane partial pressure and flow. In a second step, the partial pressure of the methane was increased to promote full surface coverage. It is important to note that for a temperature and partial pressure given, once the nuclei density was set, no significant new graphene nuclei were detected. According to this work, other groups [126] employed the same strategy during the growth process. It was clearly observed that diminishing the $\mathrm{CH}_{4}$ concentration leads to a reduction of graphene nucleation. Wu et al., [123] also developed a two-step AP CVD process with similar strategy as $\mathrm{Li}$ et al. Graphene growth was carried out at $1050 \stackrel{\circ}{\circ} \mathrm{C}$ introducing different amounts of $\mathrm{CH}_{4}$. Initially, with low $\mathrm{CH}_{4}$ concentration $(5 \mathrm{ppm})$ low nucleation density was guaranteed. After 20 min, the growth was continued by increasing $\mathrm{CH}_{4}$ concentration $(55 \mathrm{ppm}$ ) without changing any other conditions to get complete graphene coverage.

Finally, other studies [145] were developed including a two-step, AP CVD process, combining surface catalyzed process with segregation from bulk to grow bilayer graphene. Carbon atoms were firstly dissolved into the quasi-melting $\mathrm{Cu}$ metal and then segregated on the $\mathrm{Cu}$ surface to form nucleation centers.

\section{Cooling ramp}

The role of the temperature gradient during the cooling step after the synthesis process was demonstrated as a critical factor specially when working with high carbon solubility catalyst (e.g. Ni) [25]. Considering Fick's laws of diffusion, the area of FLG should dramatically increase in samples subjected to a slow cooling process. Therefore, high cooling rates, among other strategies, were commonly used to avoid (or make more difficult) carbon segregation in high solubility metals to success in the growth of high quality few layers graphene. As commented above, high quality monolayer and bilayer graphene were synthesized by using rapid processes and high cooling rates. Even when thin layers of nickel are deposited on $\mathrm{SiO}_{2} / \mathrm{Si}$ substrates to avoid the large amount of carbon diffused into nickel foils, a fast cooling rate is critical in suppressing the formation of multiple layers [26]. Unexpected results were also published where the contrary was also observed in graphene samples fabricated using a slow and fast cooling rate $(0.3$ and $3 \stackrel{\circ}{\circ} / \mathrm{s})$ [129]. A possible explanation could be that at particular experimental conditions, the surface mediated mechanism (as for the $\mathrm{Cu}$ case) plays a larger role in final film formation than the precipitation mechanism. Likely, both mechanisms will occur simultaneously during graphene growth. It 
was pointed out that the growth mechanism proposed for $\mathrm{Cu}$ systems [6], may be applicable to the $\mathrm{Ni}$ system in some cases, if the synthesis conditions are suitable to control the bulk-diffusion of the carbon into the metal to the minimum and the catalysis of the decomposition of the precursor gas is effectively achieved.

Interestingly, it was also found that the cooling rate may control the segregation behavior, strongly affecting the thickness and quality of the graphene deposit [141]. Because the concentration of carbon decreases exponentially from the surface into the bulk, an extremely fast cooling can have a quenching effect reducing the rate of carbon migration to the surface. Otherwise, extremely slow cooling rates can result in carbon with enough time to diffuse into the bulk, subsequently avoiding the carbon to segregate at the surface, strongly affecting the crystallinity. These results suggested that several layers of high quality graphene can be synthesized on $\mathrm{Ni}$ surface with optimized medium cooling rates. It was also observed that the same gas mixture should be used during the cool-down to prevent the loss of carbon from the graphene over layer (e.g., formation of $\mathrm{CO}$ and $\mathrm{CO}_{2}$ ), which can occur at temperatures higher than $300 \stackrel{\circ}{\circ}[79]$.

Published results of graphene synthesis on $\mathrm{Cu}$ also shown that high vacuum, depletion of hydrogen, and slower cooling rate $\left(18{ }^{\circ} \mathrm{C} / \mathrm{min}\right)$ compared to previous single-layer graphene synthesis can produce CVD growth of bilayer graphene[142]. It was also found that in AP CVD on $\mathrm{Cu}$, the cooling rate can significantly affect the layers number in graphene domains. High-quality monolayer graphene domains were only obtained with low cooling rates, while high cooling rates result in multilayer graphene domains with more defects, [126].

\section{SCALABILITY}

One favorable aspect of graphene synthesis by CVD is the ability to maximize the scale without losing the homogeneity of large area films, as published by Bae et al., [7]. An 8-inch-wide double tubular quartz reactor was used in this CVD system, allowing a monolayer graphene film to be synthesized on a roll of copper foil with dimensions as large as 30 inches in the diagonal direction. Excellent optical properties were measured on these films after layer-by-layer transfer to dielectric substrates, achieving bilayer, trilayer and four-layer graphene.

Roll-to-roll microwave plasma (CVD) was also used for the continuous deposition of graphene films in industrial mass production [146]. A deposition area of $294 \times 480 \mathrm{~mm}$ was demonstrated. Few layer graphene films, which consist of flakes with a nanometer size, were deposited onto a Cu foil, although it was expected that graphene films can be obtained on other substrates by optimizing the deposition conditions.

\section{BILAYER GRAPHENE}

Bilayer AB (Bernal) stacked graphene became a very interesting option in electronic applications. The stacking order and coupling in few-layer graphene were demonstrated to affect the electrical properties of the material [147]. However, a process of layer-by-layer transfer could not produce AB (Bernal) stacked graphene due to the random orientation between the transferred layers. On the other hand, by means of better control of the CVD process, bilayer graphene was grown directly on metal substrates. $[55,142,148]$ Typically, bilayer graphene was grown by feeding the growth system at higher rate of carbon or a lower rate of $\left(\mathrm{H}_{2}\right)$ but the exact growth conditions vary from one system to other due to process differences.

Lee et al., [142] reported the first synthesis of wafer scale bilayer graphene film over at least 2 in. $\times 2$ in. area, limited only by synthesis apparatus. The method was based on CVD growth of bilayer graphene on a 
copper thin film surface and was characterized by the depletion of hydrogen, high vacuum, and, most importantly, slower cooling rate compared to previous single-layer graphene synthesis. The optimal bilayer graphene film was grown at $1000{ }^{\circ} \mathrm{C}$, with growth pressure of $0.5 \mathrm{Torr}, \mathrm{CH}_{4}$ flow rate of $70 \mathrm{sccm}$, and a cooling rate of $18{ }^{\circ} \mathrm{C} / \mathrm{min}\left(0.3^{\circ} \mathrm{C} / \mathrm{s}\right)$ Characterization results confirmed highly homogeneous bilayer graphene film, with only a very small fraction corresponding to possibly three layers. The authors speculated that the key parameter for bilayer growth was the slow cooling process in comparison with publications of bilayer growth on other substrates.

More recently Bi et al., [145] developed a two-step, AP CVD process, combining surface catalyzed process with segregation from bulk to grow bilayer graphene. The yield of the bilayer graphene was demonstrated over $90 \%$. The Cu foil was firstly placed in a horizontal quartz tube and heated to $1080 \circ \mathrm{C}$ under $\mathrm{H}_{2}$ flow and the $\mathrm{CH}_{4}$ flow was secondly introduced in the chamber at $1080 \circ \mathrm{C}$. Then the furnace was switched off and cooled to a certain temperature. Finally, the $\mathrm{CH}_{4}$ flow was turned off and the $\mathrm{Cu}$ foil in the quartz tube was withdrawn for a rapid cooling. The graphene domains were prepared varying only the growth time between 3-10 min. These graphene crystallites spontaneously act as templates to induce the carbon atoms to form hexagonal bilayer graphene domains, size-tunable by controlling the growth conditions. The yield of the bilayer graphene over $90 \%$ with defect-free domains $100 \mu \mathrm{m}$ in size, was demonstrated.

\section{GRAPHENE RIBBONS AND MESHES}

Theoretical and experimental data suggest that, like bilayer graphene, a band gap could be opened in sub $10 \mathrm{~nm}$ width graphene ribbons [149]. The first synthesis of graphene nanoribbons (GNRs) was performed through mechanical exfoliation in solution. Carbon nanotubes (CNTs) were also proposed as precursors for higher yield [150]. Another interesting approach is microscopy-based patterning limited by the wavelength of light and high cost.

An interesting approach in the case of graphene nanomeshes synthesis was proposed by Bai et al., [151] by means of self-assembled colloid spheres and a metal mask deposited to fill the space between them.

\section{OTHER CONCEPTS: LASER CVD AND RAPID SYNTHESIS}

Although the CVD can achieve scalable growth of graphene using a roll-to-roll method, fabrication of graphene patterns can only be performed with the aid of expensive and time-consuming post-lithographic processes. Therefore, a convenient approach to achieve fast, scalable and affordable production of graphene patterns for electronic applications is widely required. A recent overview of laser-assisted techniques developed for fabricating carbon nanostructures, including graphene, nanotubes and nanoonions was presented by Zhou et al,. [152]. Rapid single-step fabrication of graphene patterns was achieved using laser direct writing. Line-shaped graphene ribbons of controlled widths and lengths were precisely fabricated on a $\mathrm{Ni}$ foil without extra annealing and lithographic patterning procedures. The localized laser heating resulted in a rapid thermal process at the laser beam focal point. Surprisingly, the growth rate of graphene was 1000 times faster than a conventional CVD method. Graphene patterns could be fabricated at a high scan speed up to $200 \mu \mathrm{m} / \mathrm{s}$, much faster than a traditional fabrication approach. Direct writing graphene patterns using the LCVD method could expand the capability of CVD approaches in rapid and controllable fabrication of graphene for a wide range of application. High quality of the as grown graphene was demonstrated by Raman spectroscopy. The number of graphene layers could be strictly controlled by the scan speed of the laser beam. Micron size nanoribbons with limited width were also synthesized. 


\section{SUMMARY}

An in-depth review on the synthesis of graphene by CVD techniques is presented, compiling the last more meaningful results obtained in numerous laboratories around the world. The preparation of graphene deposits necessarily requires to follow some definite steps, such as slow enough supply of carbon species to the growing surface that allows surface diffusion of carbon atoms over the substrate. Different precursors (in solid, liquid or gas forms) may be used as carbon atoms source for the preparation of graphene. Among them, a mixture hydrocarbon-hydrogen and particularly methane-hydrogen is most widely used.

Following these guidelines, firstly it is noteworthy the importance of the presence of a metal for catalysing the decomposition of precursor molecules. In this way, it is possible to control the decomposition process that supplies carbon atoms. As shown, the deposition mechanism strongly depends on the characteristics of the specific metal used as substrate/catalyst. Among these characteristics, it has to be highlighted the catalysing efficiency as well as the solubility of carbon into the metal bulk. Thereby, for metals with high carbon solubility ( $\mathrm{Ni}, \mathrm{Co} . .$. ), the main growth mechanism is the segregation and precipitation of carbon atoms located through the metal bulk immediately after the catalytic CVD process. However, for low carbon solubility metals $(\mathrm{Cu})$, the deposition mechanism is mainly dominated by the metal surface and the graphene is formed outwards from the surface. Hence, the metal used as substrate/catalyst determines the main mechanism for the graphene growth. Moreover, the characteristics of the metal surface, where the growth process takes place, result essential for the final number of layers in the graphene deposit. So, the metal substrate has to be properly treated in order to chemically reduce the metal surface and modify its structure by controlling the crystal orientation, increasing the grain size and therefore becoming smoother the metal surface.

Currently some approaches to control the number of layers of the graphene deposit via metal alloys catalysis (involving $\mathrm{Ni}$ ) were performed. There were some important advances controlling the growth of few layers varying the composition of the metal alloy catalyst, although physical, optical and electronic properties of the films still have to be necessarily improved. Also it would be highly desirable to directly growth graphene layers on dielectric substrates. More experimental work should be performed in these areas for graphene to be competitive on practical applications.

Up to now the best approach to control the monolayer growth is performed on very low solubility metal, as $\mathrm{Cu}$, since it was quite difficult to control the growth of monolayer graphene via fast cooling processes on high solubility metals (Ni). However rapid synthesis could give an opportunity to these metals. From theoretical point of view, as copper substrates are used, $\mathrm{CH}$ is the most favorable carbon species coming from methane decomposition. Subsequently, on the metal surface the energetically favorable dimmer formation from the $\mathrm{CH}$ species will occur. The dimmer formation, as well as the diffusion of the dimmer over the copper surface are dominant processes respect to the back dissociation of dimmers and the migration of carbon atoms either over the surface or towards the metal bulk. Therefore, when graphene is grown on copper foils, the main growth mechanism includes dimmers as intermediate species.

Also, it is worth to mention that, in Cu CVD graphene growth, the hexagonal lattice of $\mathrm{Cu}(111)$ favored the high quality of the as grown graphene. Moreover during the CVD heteroepitaxial growth on $\mathrm{Cu}(111)$, the orientation of graphene nuclei became well controlled with domain boundaries atomically connected. Therefore, it could be advantageous to develop techniques for producing foils with a preferential (111) surface texture. Also it has been shown that for obtaining high-uniformity graphene, it is crucial minimizing the number of imperfections, roughness and impurities, becoming the surface as uniform as possible. It is clear that initially the roughness and imperfections on the growing surface enhance graphene nucleation. However, during subsequent growth stages, the formation of a huge amount of defects is evident and thus the final deposit contains too much structural defects. Lastly, during annealing, 
the impurity atoms likely diffuse towards the Cu surface, often enhancing the decomposition of the gas precursor.

Finally, pressure and temperature are also decisive parameters for the successful growth of CVD few-layer graphene from hydrocarbon/hydrogen gas mixtures. Low pressure (LP) CVD regimes enhance evaporation of catalyst and etched domains, although the control of the number of layers seems to be more precise. It should be highlighted that the role of hydrogen remains an open question to be resolved, although etching effects were demonstrated. As expected, the partial pressure of precursor gases reveals as crucial and dependent of each reactor setup. Another significant remark is that long annealing periods of the metal substrate/catalyst at low pressure in a reducing gas combined with high temperature, leads to a low nucleation density and afterwards large domains, both facts highly desirable for high quality graphene synthesis. Lastly, low temperature processes for graphene synthesis are being developed by using plasma assisted processes. This will be an extensive area of research in the near future with the intention to synthesize controllable number of graphene layers on arbitrary substrates with a reliable and reproducible method. Also the seeded growth can be an interesting alternative for high quality and performance graphene layers with applications in several fields. Nevertheless, very high complex processes with important economical implications are involved.

\section{REFERENCES}

[1] A.K. Geim, K.S. Novoselov, Nat. Mater. 6, 183 (2007).

[2] K.S. Novoselov., Electric Field Effect in Atomically Thin Carbon Films. Sci. 306: 666-669 (2004)

[3]K. S. Novoselov, D. Jiang, F. Schedin, T. J. Booth, V. V. Khotkevich,S. V. Morozov, and A. K. Geim, Two-dimensional atomic crystals, PNAS, 102, 10451 (2005).

[4] A.K. Geim., Graphene: Status \& Prospects. Sci.324: 1530-34 (2009)

[5]P.R. Somani, S.P. Somani, and M. Umeno., Planer nanographenes from camphor by CVD, Chemical Physics Letters, 430,56 (2006)

[6] Li, $X$ et al. Large-area synthesis of high-quality and uniform graphene films on copper foils. Science 324, 1312-1314 (2009)

[7] Bae, S. et al. Roll-to-roll production of 30-inch graphene films for transparent electrodes. Nat Nanotech. 5, $574(2010)$

[8] A. H. Castro Neto, F. Guinea, N. M. R. Peres, K. S. Novoselov, A. K. Geim. The electronic properties of graphene. Rev. Mod. Phys. 81, 109 (2009)

[9] Batzill. M. The surface science of graphene: Metal interfaces, CVD synthesis, nanoribbons, chemical modifications, and defects. Surface Science Reports. 67, 83-115 (2012)

[10] Junbo Hou, Yuyan Shao, Michael W. Ellis, Robert B. Moored and Baolian Yie. Graphene-based electrochemical energy conversion and storage: fuel cells, supercapacitors and lithium ion batteries. Phys. Chem. Chem. Phys., 2011, 13, 15384-15402

[11] Xiao Huang, Xiaoying Qi, Freddy Boeyab and Hua Zhang. Graphene-based composites Chem. Soc. Rev., 2012, 41, 666-686

[12] Xiao Huang, Zongyou Yin, Shixin Wu, Xiaoying Qi , Qiyuan He, Qichun Zhang, Qingyu Yan,Freddy Boey, and Hua Zhang. Graphene-Based Materials: Synthesis, Characterization, Properties, and Applications. Small 2011, 7, No. 14, 1876-1902

[13] M. Inagaki, Y. A. Kim and M. Endo. Graphene: preparation and structural perfection. J. Mater. Chem., 2011, 21, 3280

[14] Cecilia Mattevi, Hokwon Kima and Manish Chhowalla. A review of chemical vapour deposition of graphene on copper. J. Mater. Chem. 2010, DOI: 10.1039

[15]Virendra Singh, Daeha Joung, Lei Zhai, Soumen Das, Saiful I. Khondaker, Sudipta Seal. Graphene based materials: Past, present and future. Progress in Materials Science 56 (2011) 1178-1271

[16] Wonbong Choi, Indranil Lahiri, Raghunandan Seelaboyina, and Yong Soo Kang. Synthesis of Graphene and Its Applications: A Review. Critical Reviews in Solid State and Materials Sciences, 35:52-71, 2010

[17]MOROSANU, C. E.: "Thin Films by Chemical Vapour Deposition", Elsevier

[18]SHERMAN, A.: "Chemical Vapor Deposition for Microelectronics". Noyes Publications

[19]PIERSON, H. O.: "Handbook Of Chemical Vapour Deposition". Noyes Publications,

[20]HUIMIN, L.: "Diamond Chemical Vapour Deposition". Noyes Publications

[21]RICKERBY, D. S: "Advanced Surface Coatings" . Blackie

[22]SCHUEGRAF, K. K.: "Handbook Of Thin-film Deposition Processes and Techniques", Noyes Publications

[23] Albella J.M, “Láminas Delgadas y Recubrimientos. Preparación, propiedades y aplicaciones. CSIC, 2003.

[24] Lenzsolomun P, Wu MC, Goodman W. Methane coupling at low-temperatures on Ru(0 001$)$ and Ru-11(2) bar-0 catalysts. Catal Lett 1994; $25:$ 75-86.

[25] Alfonso Reina, Xiaoting Jia, John Ho, Daniel Nezich, Hyungbin Son, Vladimir Bulovic, Mildred S. Dresselhaus and Jing Kong. Large Area, Few-Layer Graphene Films on Arbitrary Substrates by Chemical Vapor Deposition. Nanoletters 2009, 9, 30-35 
[26] Keun Soo Kim, Yue Zhao, Houk Jang, Sang Yoon Lee, Jong Min Kim, Kwang S. Kim, Jong Hyun Ahn, Philip Kim, Jae-Young Choi \& Byung Hee Hong. Large-scale pattern growth of graphene films for stretchable transparent electrodes. Nat. letters. 457, 706 (2009)

[27] Xuesong Li, Weiwei Cai, Luigi Colombo and Rodney S. Ruoff. Evolution of Graphene Growth on Ni and Cu by Carbon Isotope Labeling. NANOLETTERS 2009 Vol. 9, No. 12, 4268-4272

[28] Martin Kalbac, Otakar Frank, Ladislav Kavan. The control of graphene double-layer formation in copper catalyzed chemical vapor deposition. CARBON 50( 2012 ) 3682-3687

[29] Joseph M. Wofford, Shu Nie, Kevin F. McCarty, Norman C. Bartelt and Oscar D. Dubon Graphene Islands on Cu Foils: The Interplay between Shape, Orientation, and Defects. Nano Lett. 2010, 10, 4890-4896

[30] Wei Liu, Hong Li, Chuan Xu, Yasin Khatami, Kaustav Banerjee. Synthesis of high-quality monolayer and bilayer graphene on copper using chemical vapor deposition. CARBON 49 (2011) 4122-4130

[31] Xuesong Li, Carl W. Magnuson, Archana Venugopal, Jinho An, Ji Won Suk,Boyang Han, Mark Borysiak, Weiwei Cai, Aruna Velamakanni, Yanwu Zhu, Lianfeng Fu, Eric M. Vogel, Edgar Voelkl, Luigi Colombo,and Rodney S. Ruoff. Graphene Films with Large Domain Size by a Two-Step Chemical Vapor Deposition Process. Nano Lett. 2010, 10, 4328-4334

[32] Maria Losurdo, Maria Michela Giangregorio, Pio Capezzuto and Giovanni Bruno. Graphene CVD growth on copper and nickel: role of hydrogen in kinetics and structure. Phys. Chem. Chem. Phys., 2011, 13, 20836-20843

[33] E. M. Sacris and N. A. D. Parlee, The diffusion of hydrogen in liquid Ni, Cu, Ag, and $\mathrm{Sn}$

Metall. Mater. Trans. B, 1970, 1, 3377.

[34] W.An, X.C. Zeng and C. H.Turner, J.First-principles study of methane dehydrogenation on a bimetallic Cu/Ni111. Surface Chem. Phys., 2009, 131, 174702.

[35] Wenhua Zhang, Ping Wu, Zhenyu Li and Jinlong Yang. First-Principles Thermodynamics of Graphene Growth on Cu Surfaces. J. Phys. Chem. 2011, 115, 17782-17787

[36] Chak-Tong Au, Ching-Fai Ng, and Meng-Sheng Liao. Methane Dissociation and Syngas Formation on Ru, Os, Rh, Ir, Pd, Pt, Cu, $\mathrm{Ag}$, and Au: A Theoretical Study. Journal of Catalysis 185, 12-22 (1999)

[37] Hua Chen, Wenguang Zhu and Zhenyu Zhang. Contrasting Behavior of Carbon Nucleation in the Initial Stages of Graphene Epitaxial Growth on Stepped Metal Surfaces. PRL 104, 186101 (2010)

[38] S. Riikonen,A. V. Krasheninnikov, L. Halonen and R. M. Nieminen. The Role of Stable and Mobile Carbon Adspecies in CopperPromoted Graphene Growth. J. Phys. Chem. C 2012, 116, 5802-5809

[39] Glenn Jones, Felix Studt, Frank Abild-Pedersen, Jens K.Nørskov and Thomas Bligaard. Scaling relationships for adsorption energies of C2 hydrocarbons on transition metal surfaces. Chemical Engineering Science 66 (2011) 6318-6323

[40] J. Will Medlin and Mark D. Allendorf. Theoretical Study of the Adsorption of Acetylene on the (111) Surfaces of Pd, Pt, Ni, and Rh. J. Phys. Chem. B 2003, 107, 217-223

Cyclotrimerization Kinetics of $\mathrm{C} 2 \mathrm{H} 2$ at a Cu(110) Surface J. Phys. Chem. C 2012, 116, 9550-9560

. Adsorption and

[42] Lie-Quan Lee, Dan-Hua Shi, Yu-Jun Zhao and Pei-Lin Cao. Acetylene adsorption on Cu(111) and stepped Cu(111):theoretical study. J. Phys. Condens. Matter 7 (1995) 6449- 6457.

[43] M. Witko, K. Hermann. Different adsorbate binding mechanisms of hydrocarbons: Theoretical studies for $\mathrm{Cu}(111) \pm \mathrm{C} 2 \mathrm{H} 2 \mathrm{and}$ $\mathrm{Cu}(111) \pm \mathrm{C} 2 \mathrm{H} 4$. Applied Catalysis A: General 172 (1998) 85-95.

[44] Olaf Skibbe, Diana Vogel, Martin Binder, Annemarie Pucci, Tatyana Kravchuk, Luca Vattuone, Vinay Venugopal, Anton Kokalj and Mario Rocca. Ethene stabilization on $\mathrm{Cu}$ (111) by surface roughness. J. Chem. Phys. 131, 024701 (2009).

[45] Andrew V. Zeigarnik, Raúl E. Valdés-Pérez and Olga N. Myatkovskaya. C-C Bond Scission in Ethane Hydrogenolysis. J. Phys. Chem. B 2000, 104, 10578-10587

[46] Jeff Greeley and Manos Mavrikakis. Surface and Subsurface Hydrogen: Adsorption Properties on Transition Metals and NearSurface Alloys. J. Phys. Chem. B 2005, 109, 3460-3471

[47] Graeme Henkelman, Andri Arnaldsson and Hannes Jónsson. Theoretical calculations of $\mathrm{CH} 4$ and $\mathrm{H} 2$ associative desorption from $\mathrm{Ni}$ (111): Could subsurface hydrogen play an important role?. J. Chem. Phys. 124, 044706. 2006.

[48] Gui-Chang Wang and Junji Nakamura. Structure Sensitivity for Forward and Reverse Water-Gas Shift Reactions on Copper Surfaces: A DFT Study. J. Phys. Chem. Lett. 2010, 1, 3053-3057

[49] D. Wayne Blaylock, Teppei Ogura, William H. Green and Gregory J.O. Beran. Computational investigation of Thermochemistry and Kinetics of Steam Methane Reforming on Ni (111) under Realistic Conditions. J. Phys. Chem. C 2009, 113, 4898-4908

[50] Natasha M. Galea, Daniel Knapp, Tom Ziegler. Density functional theory studies of methane dissociation on anode catalysts in solid-oxide fuel cells: Suggestions for coke reduction. Journal of Catalysis 247 (2007) 20-33.

[51] Ping Wu, Wenhua Zhang, Zhenyu Li, Jinlong Yang and Jian Guo Hou. Communication: Coalescence of carbon atoms on Cu (111) surface: Emergence of a stable bridging-metal structure motif. J. Chem. Phys. 133, 0711012010.

[52] Neil R. Avery. Adsorption and Reactivity of Acetylene on a Cu(IIO) Surface. J. Am. Chem. SOC. 1985, 107, 6711-6712

[53] Ts.S. MARINOVA and P.K. STEFANOV. ADSORPTION AND THERMAL EVOLUTION OF ACETYLENE ON A CU(100) SURFACE. Surface Science 191 (1987) 66-74

[54] Junfeng Gao, Jijun Zhao and Feng Ding. Transition Metal Surface Passivation Induced Graphene Edge Reconstruction. J. Am. Chem. Soc. 2012, 134, 6204-6209

[55] Zhengzong Sun, Zheng Yan, Jun Yao, Elvira Beitler, Yu Zhu \& James M. Tour. Growth of graphene from solid carbon sources. 2010 | VOL 468 | Nature, 549

[56] Y. R. Luo, Comprehensive Handbook of Chemical Bond Energies, CRC Press, Boca Raton, FL 2007 , p. 9.

[57] I. Vlassiouk, M. Regmi, P. Fulvio, S. Dai, P. Datskos, G. Eres andS. Smirnov, ACS Nano, 2011, 5, 6069. 
[58] L. Gao, W. Ren, J. Zhao, L. P. Ma, Z. Chen and H. M. Cheng, Appl. Phys. Lett., 2010, 97, 183109.

[59] Xuesong Li, Carl W. Magnuson, Archana Venugopal, Rudolf M. Tromp, James B. Hannon, Eric M. Vogel, Luigi Colombo and Rodney S. Ruoff. Large-Area Graphene Single Crystals Grown by Low-Pressure Chemical Vapor Deposition of Methane on Copper. J. Am. Chem. Soc. 2011, 133, 2816-2819

[60] Ruan, G.; Sun, Z.; Peng, Z.; Tour, J. M. Growth of Graphene from Food, Insects, and Waste. ACS Nano 2011, DOI: $10.1021 / \mathrm{nn} 202625 \mathrm{c}$.

[61] Tianru Wu, Guqiao Ding, Honglie Shen, Haomin Wang, Lei Sun , Da Jiang, Xiaoming Xie and Mianheng Jiang. Triggering the Continuous Growth of Graphene Toward Millimeter-Sized Grains. Adv. Funct. Mater. 2012, DOI: 10.1002/adfm.201201577

[62] Jens Hofrichter, Bartholoma"us N. Szafranek, Martin Otto, Tim J. Echtermeyer, Matthias Baus, Anne Majerus, Viktor Geringer, Manfred Ramsteiner and Heinrich Kurz. Synthesis of Graphene on Silicon Dioxide by a Solid Carbon Source. Nano Lett. $2010,10,36-42$

[63] Reina, A.; Thiele, S.; Jia, X.; Bhaviripudi, S.; Dresselhaus, M. S.; Schaefer, J. A.; Kong, J. Growth of Large-Area Single- and BiLayer Graphene by Controlled Carbon Precipitation on Polycrystalline Ni Surfaces. Nano Res. 2009, 2, 509-516.

[64] Bin Zhang, Wi Hyoung Lee, Richard Piner, Iskandar Kholmanov, YapingWu, Huifeng Li, Hengxing Ji, and Rodney S. Ruoff. LowTemperature Chemical Vapor Deposition Growth of Graphene from Toluene on Electropolished Copper Foils. ACS Nano 2012. Published online 10.1021/nn204827h

[65] Baoshan Hu, Hiroki Ago, Carlo M. Orofeo, Yui Ogawa and Masaharu Tsujib. On the nucleation of graphene by chemical vapor deposition. New J. Chem., 2012, 36, 73-77

[66] K. S. Kim, Y. Zhao, H. Jang, S. Y. Lee, J. M. Kim, K. S. Kim, J.-H. Ahn, P. Kim, J.-Y. Choi and B. H. Hong, Nature, 2009, $457,706$.

[67] S.-Y. Kwon, C. V. Ciobanu, V. Petrova, V. B. Shenoy, J. Bare no,V. Gambin, I. Petrov and S. Kodambaka, Nano Lett., 2009, 9, 3985.

[68] P. W. Sutter, J.-I. Flege and E. A. Sutter, Nat. Mater., 2008, 7, 406.

[69] J. Coraux, A. T. N'Diaye, C. Busse and T. Michely, Nano Lett., 2008,8, 565.

[70] E. Loginova, N. C. Bartelt, P. J. Feibelman and K. F. McCarty.Factors influencing graphene growth on metal Surfaces.New Journal of Physics 11 (2009) 063046 (20pp)

[71] Libo Gao, Wencai Ren, Huilong Xu, Li Jin, Zhenxing Wang, Teng Ma, Lai-Peng Zhiyong Zhang, Qiang Fu, Lian-Mao Peng, Xinhe Bao \& Hui-Ming Cheng. Repeated growth and bubbling transfer of graphene with millimetre-size single-crystal grains using platinum. Nat. Communications |3:699| Dol: 10.1038/ncomms1702

[72]A. Earnshaw; T. J. Harrington. The chemistry of the transition elements; Oxford University Press, 1972.

[73] Junbo Hou, Yuyan Shao, Michael W. Ellis, Robert B. Moored and Baolian Yie. Graphene-based electrochemical energy conversion and storage: fuel cells, supercapacitors and lithium ion batteries. Phys. Chem. Chem. Phys., 2011, 13, 15384-15402

[74] A. N. Obraztsov, E. A. Obraztsova, A. V. Tyurnina and A. A. Zolotukhin, Carbon, 2007, 45, 2017.

[75] A. Reina, X. Jia, J. Ho, D. Nezich, H. Son, V. Bulovic,M. S. Dresselhaus and J. Kong, Nano Lett., 2009, 9, 30.

[76] Yunzhou Xue, Bin Wu, Yunlong Guo, Liping Huang, Lang Jiang, Jianyi Chen, Dechao Geng, Yunqi Liu, Wenping Hu, and Gui Yu Synthesis of Large-Area, Few-Layer Graphene on Iron Foil by Chemical Vapor Deposition Nano Res. 2011, 4(12): 1208-1214

[77] Maxwell Zheng, Kuniharu Takei, Benjamin Hsia, Hui Fang, Xiaobo Zhang,Nicola Ferralis, Hyunhyub Ko, Yu-Lun Chueh, Yuegang Zhang, Roya Maboudian, and Ali Javey. Metal-catalyzed crystallization of amorphous carbon to graphene. APPLIED PHYSICS LETTERS 96, 063110, 2010.

[78] Shanshan Chen, Weiwei Cai, Richard D. Piner, Ji Won Suk, Yaping Wu, Yujie Ren, Junyong Kang and Rodney S. Ruoff. Synthesis and Characterization of Large-Area Graphene and Graphite Films on Commercial Cu-Ni Alloy Foils. Nano Lett. 2011, 11, 3519-3525

[79] Zachary R. Robinson, Parul Tyagi, Thomas M. Murray, and Carl A. Ventrice Jr, Shanshan Chen, Andrew Munson, Carl W. Magnuson, and Rodney S. Ruoff. Substrate grain size and orientation of $\mathrm{Cu}$ and $\mathrm{Cu}$-Ni foils used for the growth of graphene films. J. Vac. Sci. Technol. A 30(1), Jan/Feb 2012

[80] T. Sakurai, T. Hashizume, A. Kobayashi, A. Sakai, S. Hyodo, Y. Kuk. And H. W. Pickering, Phys. Rev. B 34, 8379 (1986).

[81] Xun Liu, Lei Fu, Nan Liu, Teng Gao, Yanfeng Zhang, Lei Liao, and Zhongfan Liu. Segregation Growth of Graphene on Cu-Ni Alloy for Precise Layer Control. J. Phys. Chem. C 2011, 115, 11976-11982

[82] Robert S. Weatherup, Bernhard C. Bayer, Raoul Blume, Caterina Ducati, Carsten Baehtz, Robert Schlogl, and Stephan Hofmann. In Situ Characterization of Alloy Catalysts for Low-Temperature Graphene Growth. Nano Lett. 2011, 11, 4154-4160

[83] S. K. Jerng, D. S. Yu, Y. S. Kim, J. Ryou, S. Hong, C. Kim, S. Yoon, D. K. Efetov, P. Kim and S. H. Chun, J. Phys. Chem. C, 2011, $115,4491-4494$.

[84] K. B. Kim, C. M. Lee and J. Choi, J. Phys. Chem. C, 2011, 115, 14488-14493.

[85] C. M. Lee and J. Choi, Appl. Phys. Lett., 2011, 98, 183106.

[86] L. Zhang, Z. Shi, Y. Wang, R. Yang, D. Shi and G. Zhang, Nano Res., 2011, 4, 315-321.

[87] X. Ding, G. Ding, X. Xie, F. Huang and M. Jiang, Carbon, 2011, 49, 2522-2525.

[88] M. H. Rummeli, A. Bachmatiuk, A. Scott, F. Borrnert, J. H. Warner, V. Hoffman, J. H. Lin, G. Cuniberti and B. B€uchner, ACS Nano, 2010, 4, 4206-4210.

[89] H. Bi, F. Q. Huang, J. Liang, X. M. Xie and M. H. Jiang, Adv. Mater., 2011, 23, 3202-3206.

[90] Hui Bi, Shengrui Sun, Fuqiang Huang, Xiaoming Xieb and Mianheng Jiang. Direct growth of few-layer graphene films on $\mathrm{SiO}_{2}$ substrates and their photovoltaic applications. J. Mater. Chem., 2012, 22, 411

[91] Ariel Ismach, Clara Druzgalski, Samuel Penwell, Adam Schwartzberg, Maxwell Zheng, Ali Javey, Jeffrey Bokor and Yuegang Zhang. Direct Chemical Vapor Deposition of Graphene on Dielectric Surfaces. Nano Lett. 2010, 10, 1542-1548 
[92] Kwanpyo Kim, Zonghoon Lee, William Regan,C. Kisielowski, M. F. Crommie and A. Zettl. Grain Boundary Mapping in Polycrystalline Graphene. ACS Nano, VOL. 5 NO. 3 2142-2146 2011

[93] Li Gao, Jeffrey R. Guest and Nathan P. Guisinger. Epitaxial Graphene on Cu(111). Nano Lett. 2010, 10, 3512-3516

[94] L. Zhao, K.T. Rim, H. Zhou, R. He, T.F. Heinz, A. Pinczuk, G.W. Flynn, A.N. Pasupathy Influence of copper crystal surface on the CVD growth of large area monolayer graphene. Solid State Communications 151 (2011) 509-513

[95]Joshua D. Wood, Scott W. Schmucker, Austin S. Lyons, Eric Pop and Joseph W. Lyding. Effects of Polycrystalline Cu Substrate on Graphene Growth by Chemical Vapor Deposition. Nano Lett. 2011, 11, 4547-4554

[96] Zhang, W.; Wu, P.; Li, Z.; Yang, J. J. Phys. Chem. C 2011, 115 (36) 17782-17787.

[97] Chatain, D.; Ghetta, V.; Wynblatt, P. Interface Sci. 2004, 12 (1), 7-18.

[98] Ago, H.; Ito, Y.; Mizuta, N.; Yoshida, K.; Hu, B.; Orofeo, C. M.; Tsuji, M.; Ikeda, K.; Mizuno, S. Epitaxial Chemical Vapor Deposition Growth of Single-Layer Graphene over Cobalt Film Crystallized on Sapphire. ACS Nano 2010, 4, 7407-7414.

[99] Iwasaki, T.; Park, H. J.; Konuma, M.; Lee, D. S.; Smet, J. H.; Starke, U. Long-Range Ordered Single-Crystal Graphene on HighQuality Heteroepitaxial Ni Thin Films Grown on MgO(111). Nano Lett. 2011, 11, 79-84.

[100] Hu, B.; Ago, H.; Ito, Y.; Kawahara, K.; Tsuji, M.; Magome, E.; Sumitani, K.; Mizuta, N.; Ikeda, K.; Mizuno, S. Epitaxial Growth of Large-Area Single-Layer Graphene over Cu(111)/sapphire by Atmospheric Pressure CVD. Carbon 2012, 50, 57-65.

[101] Reddy, K. M.; Gledhill, A. D.; Chen, C. H.; Drexler, J. M.; Padturea, N. P. High Quality, Transferrable Graphene Grown on Single Crystal Cu(111) Thin Films on Basal-Plane Sapphire. Appl. Phys. Lett. 2011, 98, 113117.

[102] Van, C. V.; Kimouche, A.; Plantey, A. R.; Fruchart, O.; Guillemaud, P. B.; Bendiab, N.; Coraux, J. Epitaxial Graphene Prepared by Chemical Vapor Deposition on Single Crystal Thin Iridium Films on Sapphire. Appl. Phys. Lett. 2011, 98, 181903.

[103] Sutter, P. W.; Albrecht, P. M.; Sutter, E. A. Graphene Growth on Epitaxial Ru Thin Films on Sapphire. Appl. Phys. Lett. 2010, $97,213101$.

[104] Yoshii, S.; Nozawa, K.; Toyoda, K.; Matsukawa, N.; Odagawa, A.; Tsujimura, A. Suppression of Inhomogeneous Segregation in Graphene Growth on Epitaxial Metal Films. Nano Lett. 2011, 11, 2628-2633.

[105] Yui Ogawa, Baoshan Hu, Carlo M. Orofeo, Masaharu Tsuji, Ken-ichi Ikeda, Seigi Mizuno, Hiroki Hibino and Hiroki Ago. Domain Structure and Boundary in Single-Layer Graphene Grown on $\mathrm{Cu}(111)$ and $\mathrm{Cu}(100)$ Films. J. Phys. Chem.Lett. 2012, 3, 219-226

[106] Mark P. Levendorf, Carlos S. Ruiz-Vargas, Shivank Garg, and Jiwoong Park. Transfer-Free Batch Fabrication of Single Layer Graphene Transistors. Nano Lett., Vol. 9, No. 12, 2009

[107] Casey A Howsare, XiaojunWeng, Vince Bojan, David Snyder and Joshua A Robinson. Substrate considerations for graphene synthesis on thin copper films. Nanotechnology 23 (2012) 135601

[108] Jakub Kedzierski, Pei-Lan Hsu, Alfonso Reina, Jing Kong, Paul Healey, Peter Wyatt, and Craig Keast. Graphene-on-Insulator Transistors Made Using C on Ni Chemical-Vapor Deposition. IEEE ELECTRON DEVICE LETTERS, VOL. 30, NO. 7, JULY 2009

[109] Zhengtang Luo, Ye Lu, Daniel W. Singer, Matthew E. Berck, Luke A. Somers, Brett R. Goldsmith and A. T. Charlie Johnson. Effect of Substrate Roughness and Feedstock Concentration on Growth of Wafer-Scale Graphene at Atmospheric Pressure. Chem. Mater. 2011, 23, 1441-1447

[110] Chen, H.; Zhu, W.; Zhang, Z. Phys. Rev. Lett. 2010, 104, 186101.

[111] Souheil Saadi, Frank Abild-Pedersen, Stig Helveg, Jens Sehested, Berit Hinnemann, Charlotte C. Appel and Jens K. Nørskov. On the Role of Metal Step-Edges in Graphene Growth. J. Phys. Chem. C 2010, 114, 11221-11227

[112] Helveg, S.; Lope'z-Cartes, C-; Sehested., J.; Hansen., P. L.; Clausen, B. S.; Rostrup-Nielsen, J. R.; Abild-Pedersen, F.; Nørskov, J. K. Nature 2004, $427,426$.

[113] Abild-Pedersen, F.; Nørskov, J. K.; Rostrup-Nielsen, J. R.; Sehested, S.; Helveg, S. Phys. ReV. B 2006, 73, 115419.

[114] Bengaard, H.; Nørskov, J. K.; Sehested, J.; Clausen, B. S.; Nielsen, L. P.; Molenbroek, A. M.; Rostrup-Nielsen, J. R. J. Catal. 2002, 209, 365

[115] Shu Nie, Joseph M. Wofford, Norman C. Bartelt, Oscar D. Dubon, and Kevin F. McCarty. Origin of the mosaicity in graphene grown on Cu(111). PHYSICAL REVIEW B 84, 155425 (2011)

[116]E. Sutter, P. Albrecht, and P. Sutter, Graphene growth on polycrystalline Ru thin films Appl. Phys. Lett. 95 , 133109 (2009).

[117] Pinshane Y. Huang, Carlos S. Ruiz-Vargas, Arend M. van der Zande, William S. Whitney, Mark P. Levendorf, Joshua W. Kevek, Shivank Garg, Jonathan S. Alden, Caleb J. Hustedt5, Ye Zhu, Jiwoong Park, Paul L. McEuen \& David A. Muller. Grains and grain boundaries in single-layer graphene atomic patchwork quilts. 20 J A N U A RY 2011 | VO L 469 | N AT U RE | 391

[118] F. Jansen, M. A. Machonkin, D. E. Kuhman, J. Vac. Sci. Technol. A-Vac. Surf. Films 1990, 8, 3785 .

[119] Q. K. Yu , L. A. Jauregui , W. Wu , R. Colby , J. F. Tian , Z. H. Su , H. L. Cao , Z. H. Liu , D. Pandey , D. G. Wei , T. F. Chung , P. Peng, N. Guisinger, E. A. Stach , J. M. Bao, S. S. Pei ,Y. P. Chen. Control and characterization of individual grains and grain boundaries in graphene grown by chemical vapour deposition Nat. Mater. 2011, 10, 443 .

[120] Wei Wu , Luis A. Jauregui , Zhihua Su, Zhihong Liu , Jiming Bao, Yong P. Chen and Qingkai Yu. Growth of Single Crystal Graphene Arrays by Locally Controlling Nucleation on Polycrystalline Cu Using Chemical Vapor Deposition. Adv. Mater. 2011, 23, 4898-4903

[121] Sreekar Bhaviripudi, Xiaoting Jia, Mildred S. Dresselhaus, and Jing Kong. Role of Kinetic Factors in Chemical Vapor Deposition Synthesis of Uniform Large Area Graphene Using Copper Catalyst. Nano Lett. 2010, 10, 4128-4133

[122] Alex W. Robertson and Jamie H. Warner Hexagonal Single Crystal Domains of Few-Layer Graphene on Copper Foils. Nano Lett. 2011, 11, 1182-1189

[123] Wei Wu, Qingkai Yu, Peng Peng, Zhihong Liu, Jiming Bao and Shin-Shem Pei. Control of thickness uniformity and grain size in graphene films for transparent conductive electrodes. Nanotechnology 23 (2012) 035603 
[124] Tommi Kaplas, Deepika Sharma, Yuri Svirko. Few-layer graphene synthesis on a dielectric substrate. CARBON 50 (2012) $1503-1509$

[125] Yi Zhang, Luyao Zhang, Pyojae Kim, Mingyuan Ge, Zhen Li and Chongwu Zhou. Vapor Trapping Growth of Single-Crystalline Graphene Flowers: Synthesis, Morphology, and Electronic Properties dx.doi.org/10.1021/nl300039a | Nano Lett.

[126] Hong Wang, Guanzhong Wang, Pengfei Bao, Shaolin Yang, Wei Zhu, Xing Xie and Wen-Jun Zhang. Controllable Synthesis of Submillimeter Single-Crystal Monolayer Graphene Domains on Copper Foils by Suppressing Nucleation. J. Am. Chem. Soc. 2012, $134,3627-3630$

[127] Zhang, Y.; Li, Z.; Kim, P.; Zhang, L.; Zhou, C. ACS Nano 2012, 6, 126.

[128] L. Huang, Q.H. Chang, G.L. Guo, Y. Liu, Y.Q. Xie, T. Wang, B. Ling, H.F. Yang. Synthesis of high-quality graphene films on nickel foils by rapid thermal chemical vapor deposition. CA R B O N 50 ( 2012 ) $551-556$

[129] Juang ZY, Wu CY, Lu AY, Su CY, Leou KC, Chen FR, et al. Graphene synthesis by chemical vapor deposition and transfer by a roll-to-roll process. Carbon 2010;48:3169-74.

[130] Park HJ, Meyer J, Roth S, Ska'kalova' V. Growth and properties of few-layer graphene prepared by chemical vapor deposition. Carbon 2010;48:1088-94.

[131] Gao L, Ren W, Zhao J, Ma LP, Chen Z, Cheng HM. Efficient growth of high-quality graphene films on Cu foils by ambient pressure chemical vapor deposition. Appl Phys Lett

2010;97:183109-1-3.

[132] Dechao Geng, Bin Wu, Yunlong Guo, Liping Huang, Yunzhou Xue, Jianyi Chen, Gui Yu, Lang Jiang, Wenping Hu, and Yunqi Liu. Uniform hexagonal graphene flakes and films grown on liquid copper surface. 7992-7996. PNAS . May 22, 2012. vol. 109.no. 21

[133] Wu B, et al. Equiangular hexagon-shape-controlled synthesis of graphene on copper surface. Adv Mater 23:3522-3525. (2011)

[134] K. S. Novoselov, V. I. Falko, L. Colombo, P. R. Gellert, M. G. Schwab \& K. Kim. A roadmap for graphene. 192|N AT U R E| VOL 490 | 11 OCTOBER 2012

[135] Jaeho Kim, Masatou Ishihara, Yoshinori Koga, Kazuo Tsugawa, Masataka Hasegawa, and Sumio lijima. Low-temperature synthesis of large-area graphene-based transparent conductive films using surface wave plasma chemical vapor deposition. APPLIED PHYSICS LETTERS 98, 091502,2011

[136] Y. Kim, W. Song, S. Y. Lee, C. Jeon, W. Jung, M. Kim, and C.-Y. Park. Low-temperature synthesis of graphene on nickel foil by microwave plasma chemical vapor deposition. APPLIED PHYSICS LETTERS 98, 263106 (2011)

[137] Tomo-o Terasawa, Koichiro Saiki. Growth of graphene on Cu by plasma enhanced chemical vapor deposition. CARBON 50 ( 2012) 869-874.

[138] Qi J L, Zhang L X, Cao J, et al. Synthesis of graphene on a Ni film by radio-frequency plasma-enhanced chemical vapor deposition. Chin Sci Bull, doi: 10.1007/s11434-012-5120-4

[139] Golap Kalita, Madhu Sudan Kayastha, Hideo Uchida, Koichi Wakita and Masayoshi Umeno. Direct growth of nanographene films by surface wave plasma chemical vapor deposition and their application in photovoltaic devices. RSC Advances, 2012, 2, 3225-3230.

[140] A. Kumar, A.A. Voevodin, D. Zemlyanov, D.N. Zakharov, T.S. Fisher. Rapid synthesis of few-layer graphene over Cu foil. CARBON 50 (2012) 1546-1553.

[141] Qingkai Yu, Jie Lian, Sujitra Siriponglert, Hao Li, Yong P. Chen and Shin-Shem Pei. Graphene segregated on Ni surfaces and transferred to insulators. APPLIED PHYSICS LETTERS 93, 113103.2008.

[142] Seunghyun Lee, Kyunghoon Lee and Zhaohui Zhong. Wafer Scale Homogeneous Bilayer Graphene Films by Chemical Vapor Deposition. Nano Lett. 2010, 10, 4702-4707

[143] Gao, J.; Yip, J.; Zhao, J.; Yakobson, B. I.; Ding, F. J. Am. Chem. Soc. 2011, 133, 5009.

[144] H. H. Angermann and Z. Horz, Appl. Surf. Sci. 70, 163, 1993.

[145] Hui Bi, Fuqiang Huang, Wei Zhao, Xujie Lu“, Jian Chen, Tianquan Lin, Dongyun Wan, Xiaoming Xie, Mianheng Jiang. The production of large bilayer hexagonal graphene domains by a two-step growth process of segregation and surface catalytic chemical vapor deposition. CARBON 50 (2012) 2703-2709

[146] Takatoshi Yamada, Masatou Ishihara, Jaeho Kim, Masataka Hasegawa, Sumio lijima. A roll-to-roll microwave plasma chemical vapor deposition process for the production of $294 \mathrm{~mm}$ width graphene films at low temperatura. CARBON 50 (2012) 2615-2619

[147] Ohta, T.; Bostwick, A.; Seyller, T.; Horn, K.; Rotenberg, E. Controlling the Electronic Structure of Bilayer Graphene. Science 2006, 313, 951-954.

[148] Yan, K.; Peng, H.; Zhou, Y.; Li, H.; Liu, Z. Formation of Bilayer Bernal Graphene: Layer-by-Layer Epitaxy by Chemical Vapor Deposition. Nano Lett. 2011, 11, 1106-1110.

[149] Li, X.; Wang, X.; Zhang, L.; Lee, S.; Dai, H. Chemically Derived. Ultrasmooth Graphene Nanoribbon Semiconductors. Science 2008, 319, 1229-1232.

[150] Kosynkin, D. V.; Lu, W.; Sinitskii, A.; Pera, G.; Sun, Z.; Tour, J. M. Highly Conductive Graphene Nanoribbons by Longitudinal Splitting of Carbon Nanotubes using Potassium Vapor. ACS Nano 2011, 5, 968-974.

[151] Bai, J.; Zhong, X.; Jiang, S.; Huang, Y.; Duan, X. Graphene Nanomesh. Nat. Nanotechnol 2010, 5, 190-194.

[152] Yun Shen Zhou, Wei Xiong, Jongbok Park, Min Qian, Masoud Mahjouri-Samani, Yang Gao, Lan Jiang, Yongfeng Lu. Laserassisted nanofabrication of carbon nanostructures. J. Laser Appl., Vol. 24, No. 4, July 2012 